Review Article

\title{
Theoretical Simulations of Reactive and Nonreactive Scattering of Light Diatomic Molecules from Metal Surfaces: Past, Present, and Future
}

\author{
C. Díaz \\ Departamento de Química Módulo 13, Universidad Autónoma de Madrid, 28049 Madrid, Spain \\ Correspondence should be addressed to C. Díaz; cristina.diaz@uam.es
}

Received 15 May 2014; Accepted 10 July 2014; Published 10 September 2014

Academic Editor: Qiaohui Fan

Copyright (C) 2014 C. Díaz. This is an open access article distributed under the Creative Commons Attribution License, which permits unrestricted use, distribution, and reproduction in any medium, provided the original work is properly cited.

\begin{abstract}
In everyday life we are surrounded by surfaces and, therefore, by phenomena involving molecule-surface interactions. Furthermore, the processes of heterogeneous catalysis, which are governed by molecule-surface interactions, are of huge practical importance, because the production of most synthetic compounds involves catalytic processes, which explains the tremendous effort that surface science scientists have invested to understand the basic principles underlying elementary interactions between light molecules and surfaces. This effort was recognized in 2007 with the Nobel prize in chemistry awarded to Gerhard Ertl. Here we revise some of the most relevant studies performed so far in this field. We also point out the major challenges that the surface science community may face in this field in the years to come.
\end{abstract}

\section{Introduction}

In everyday life we are surrounded by phenomena involving molecule-surface interactions. For example, the corrosion of a coin is due to the interaction between the oxygen molecules in the air and the metal surface atoms, which cause a structural damage leaving a layer of oxidized material (rust) on the coin. Another example is the green appearance of the domes of some buildings, which is due to the oxidation of copper, material from which domes are made. Atomic and molecular interactions on surfaces also play a key role in many industrial processes, such as corrosion, friction, lubrication, oxidation, hydrogen storage, and heterogeneous catalysis. Heterogeneous catalysis is of tremendous practical importance. The production of most synthetic compounds involves catalytic processes because most of the chemical reactions relevant to chemical industries are too slow in the absence of a catalyst. Therefore, understanding the basic principles that govern the geometry and electronic structure of metal surfaces and the elemental processes occurring on them, such as molecular reactivity and molecular scattering, has been and still is one major scope in surface science. At this point, it should be noticed that the importance of this research field was recognized in 2007 with the Nobel prize in chemistry awarded to Professor Ertl for the detailed description of the sequence of elementary molecule-surface reactions by which vast quantities of ammonia are produced [1]. Ammonia production is basic for the fertilizers industry.

Metal surfaces serve as catalysts for many chemical reactions. Some of these reactions, more efficient on a metal surface than in the gas phase, are, for example, hydrogenation of $\mathrm{O}, \mathrm{C}, \mathrm{N}$, and $\mathrm{S}$ to obtain $\mathrm{H}_{2} \mathrm{O}, \mathrm{CH}_{4}, \mathrm{NH}_{3}$, and $\mathrm{H}_{2} \mathrm{~S}$; oxidation of ammonia to nitric acid, which is a basic reaction in the production of fertilizers; methanol synthesis from $\mathrm{CO}$ and $\mathrm{H}_{2}$; oxidation of ethylene to ethylene oxide, a basic reaction in the production of antifreezes; and dehydrogenation of butane to butadiene, a reaction of primary importance in the production of synthetic rubber.

A detailed knowledge, at atomic scale, of the dynamic processes that govern the molecular reactions on surfaces is essential to design and develop new and improved catalysts. In this regard, detailed theoretical studies of these kinds of processes are of most fundamental interest. Theoretical simulations are used not only to provide accurate insights into experimental results, but also to predict new trends. They can be used to decrease the number of trial and 


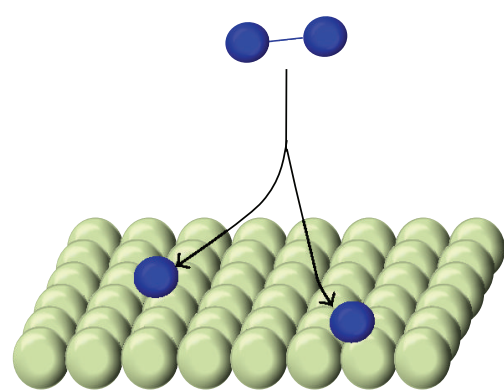

(a)

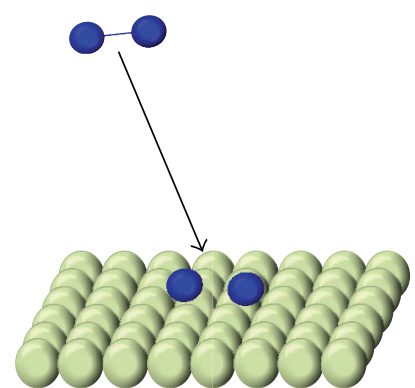

(b)

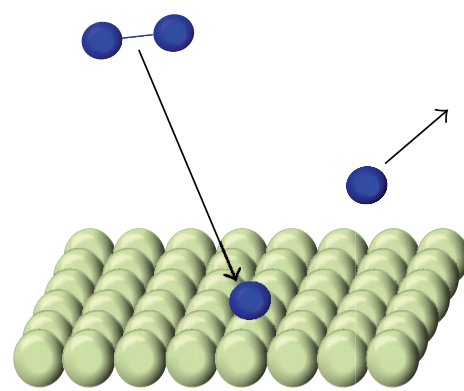

(c)

FIGURE 1: (a) dissociative adsortion mechanism; (b) molecular adsorption mechanism; and (c) abstraction mechanism.

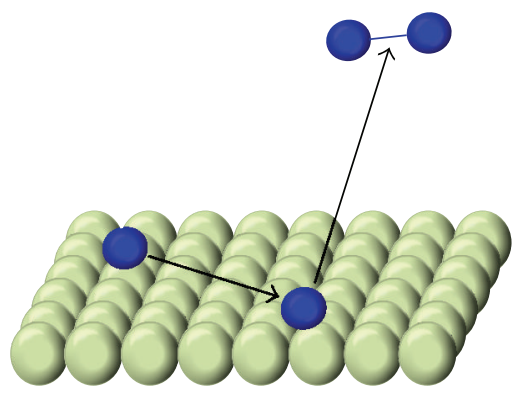

(a)

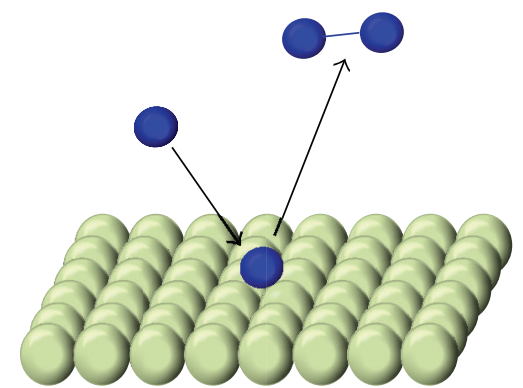

(b)

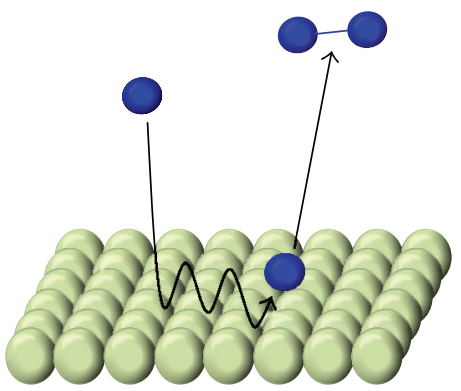

(c)

FIGURE 2: Schematic representation of the (a) Langmuir-Hinshelwood mechanism; (b) Eley-Rideal mechanism; and (c) hot-atom mechanism.

error experiments conventionally used in the design of new devices. For example, five chemical compounds could be combined in thousands of different ways to build a catalyst; the use of theoretical simulations can reduce the number of experiments needed to optimize the structure of this catalyst.

A wealth of chemical and physical processes, involving light molecules, are possible on surfaces. These processes can be divided into three main groups.

(1) Molecular adsorption (see Figure 1): it is a physical process in which a molecule coming from the vacuum (gas phase) hits a surface somehow and sticks on it. This process may induce a relatively high concentration of molecules (or atoms) at the place of contact, and, therefore, to the formation of a molecular or atomic film on the surface. Four primary physical mechanisms are associated with the molecular adsorption mechanism:

(a) molecular physisorption: a molecule, coming from the vacuum, gets adsorbed on the surface and weakly binds to it, due to van der Waals forces;

(b) molecular chemisorption: a molecule, coming from the vacuum, gets adsorbed on the surface and strongly binds to it, due to the formation of new chemical bonds;

(c) dissociative chemisorption: a molecule, coming from the vacuum, breaks its bond and its atoms get adsorbed on the surface thanks to the formation of new chemical bonds;

(d) abstraction mechanism: a molecule, coming from the gas phase, breaks its bond; one of its atoms gets absorbed on the surface and the other one escapes back to the vacuum.

(2) Molecular desorption (see Figure 2): it is the opposite process to the molecular adsorption one. In this case a molecule previously absorbed on the surface is released. Physical mechanisms associated with molecular desorption are

(a) Langmuir-Hinshelwood: two atoms or molecules adsorbed on a surface, in thermal equilibrium with it, meet each other and react; as a result a new molecule is formed and leaves the surface;

(b) Eley-Rideal: an atom coming from the vacuum reacts with an atom adsorbed on the surface (in thermal equilibrium with it) forming a new molecule, which desorbs from the surface;

(c) Hot-atom mechanism: a reaction takes place between an adsorbed atom, in thermal equilibrium with the surface, and an atom that has recently arrived from the vacuum, which is not in thermal equilibrium yet.

(3) Molecular scattering (see Figure 3): a molecule, coming from the vacuum, collides with the surface and 


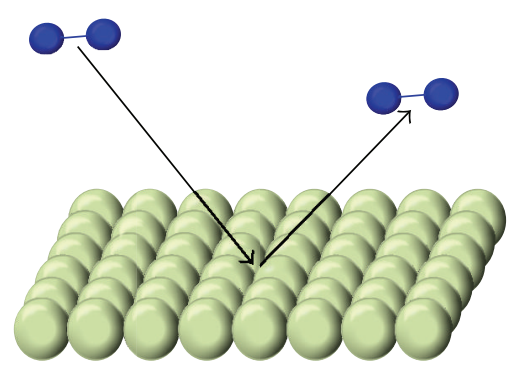

(a)

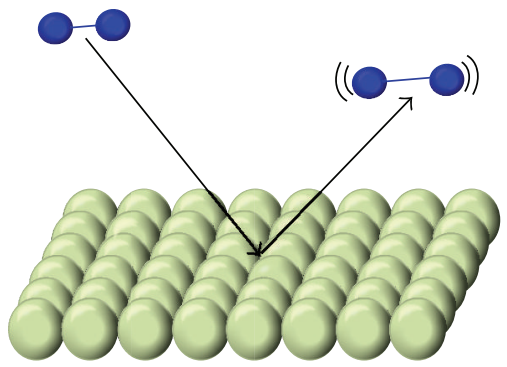

(c)

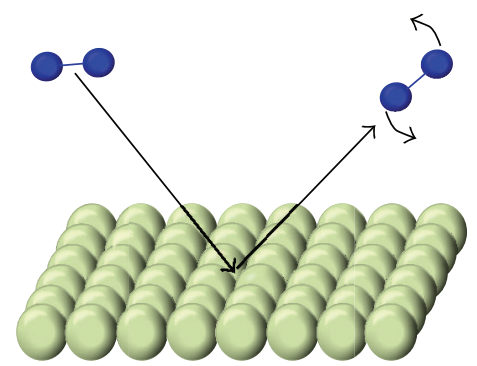

(b)

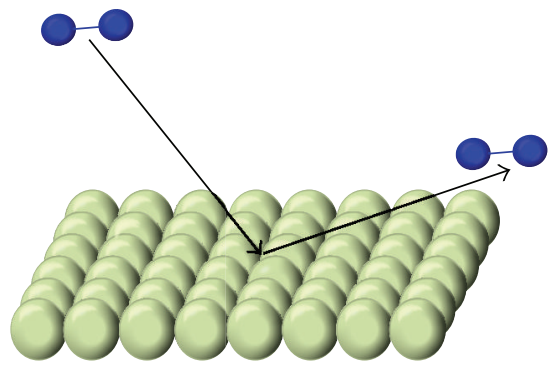

(d)

FIGURE 3: Schematic representation of the (a) elastic scattering mechanism; (b) rotational excitation mechanism; (c) vibrational excitation mechanism; and (d) diffraction mechanism.

is reflected back to it. During this physical process the molecule can transfer (gain) energy to (from) the surface. Based on this energy transfer we can distinguish the following phenomena:

(a) elastic scattering: there is not transfer of energy between the molecule and the surface during the collision;

(b) vibrationally inelastic scattering: the molecular vibrational energy increases or decreases during the collision, due to an energy transfer towards (from) the molecular vibrational motion from (toward) the surface;

(c) rotationally inelastic scattering: the molecular rotational energy increases or decreases during the collision, due to an energy transfer towards (from) the molecular rotational motion from (towards) the surface;

(d) diffraction: the parallel (to the surface) momentum of the molecule changes by discrete quantities, due to the periodicity of the surface; as a result the angular distribution of the scattered molecule presents a discrete peaks distribution.

In this review we will focus mainly on three of these mechanisms: (i) dissociative chemisorption [2,3]; (ii) molecular scattering; and (iii) diffraction [4-6].

\section{Experimental Techniques in Brief}

Molecule-surface interactions experiments are, in general, carried out in ultrahigh vacuum systems (see [7] and references therein). These systems are formed by a number of connected chambers, which can be individually pumped. They contain a number of skimmers and orifices that are used to create a supersonic molecular beam (SMB) from an initial pulsed nozzle source, at high stagnation pressure. These SMBs are well defined by its stream velocity and the width of its velocity distribution. The average translational energy of the molecular beam can be varied by heating or cooling the nozzle.

SMB experiments can be used to measure the dissociative adsorption probability of a molecule-surface system using the so-called King and Wells' method [8]. In this method, the partial pressure of the molecular gas is monitored as a function of time using a quadrupole mass spectrometer (QMS). At the beginning of the measurement the supersonic beam of molecules is produced in a secondary chamber equipped with a shutter, which can intercept the beam. While the shutter intercepts the molecular beam, the QMS, located in the main chamber, measures the background signal $P_{0}$. Once the shutter is turned down, the beam enters the main chamber increasing the signal measured by the QMS to $P_{1}$. In the main chamber a second shutter intercepts the beam preventing it from striking the surface target. When this second shutter is removed from the path of the beam, the molecules can hit and stick on the surface. Whenever adsorption takes place the QMS signal decreases as $P(t)$. Thus, the relative decrease of the QMS signal gives us the absolute dissociation probability

$$
R(t)=\frac{P_{1}-P(t)}{P_{1}-P_{0}} .
$$

The dissociative adsorption probability can be also measured using temperature-programmed desorption (TPD) 
techniques (see, e.g., [9]). In this case, the molecules, previously adsorbed on a surface, are desorbed by heating the surface and simultaneously are detected by a mass spectrometer. In these kinds of experiments, the adsorption probabilities are obtained from coverage versus exposure measurements. Thus, the adsorption probability is determined directly as the ratio of the number of molecules that adsorb to the number of molecules that strike the surface. The number of the adsorbed molecules is proportional to the area under the TPD trace. The reliability of the TPD technique lies in the validity of the detailed balance principle [10], which assumes that the dissociation probability can be measured by looking at the reverse process, the molecular desorption.

Associative desorption measurements are also performed using time-of-flight (TOF) techniques [11], which allow obtaining molecular-state-specific probabilities. In general, TOF experiments are performed on a two-chamber apparatus. One of the chambers contains the crystal into which the molecules are adsorbed after permeation through the bulk. The molecules desorbing from the surface are then probed by laser ionization detection in the second chamber. And the TOF distributions are obtained by recording the time of flight of the photoions to a multichannel plate detector. To determine quantum state distributions, the relative signal intensity for each quantum state desorbed from the surface is compared with the corresponding signal obtained with an effusive Knudsen source of molecules.

TOF experiments are also used to measure rotational and vibrational inelastic scattering probabilities, in combination with stimulated Raman pumping (SRP) [12] and resonance enhanced multiphoton ionization (REMPI) techniques. SRP can be used to excite vibrationally the initial molecular beam, thus selecting the initial vibrational state. For this aim, the initial molecular beam is crossed with two focused laser beams, which are chosen in such a way that the frequency difference between them matches the vibration of the molecule, allowing an efficient excitation of the molecules from the ground state to an excited state. The rotational and vibrational populations as well as the quadrupole alignment of the scattered molecules can be determined using REMPI $[13,14]$. In applying this technique, the probe laser beam is focused on the molecular beam few $\mathrm{mm}$ in front of the surface where it ionizes the molecules, and the ions are collected and detected with a microchannel electron multiplier plate.

SMB techniques can also be used to measure diffractive scattering probabilities [15]. In these kinds of experiments the monochromatic character of the molecular beams plays a crucial role; spread-velocity beams do not allow observing diffraction peaks. To measure diffraction from SMB experiments two types of apparatus are commonly used: (i) in the fixed-angle setups, the angle between the incident and the reflected beam is fixed; that is, $\Theta_{i}+\Theta_{f}=$ const, and the angular distributions of the diffracted particles are measured by rotating continuously the crystal and thus the incident angle $\Theta_{i}$ varies during data acquisition; (ii) in the rotary setups, the detector is able to rotate around the crystal from a given incidence angle. Therefore, the diffraction beam can be measured in a large region of the reciprocal space. The advantage of this latter setup is the possibility of determining absolute diffraction probabilities.

\section{Molecule-Surface Dynamics Simulations}

Since the early 90 s the interactions of light molecules with surfaces have received more and more attention from theoretical surface scientists, essentially due to the development of multidimensional quantum dynamics methods $[2,3,16,17]$ and the development of interpolation methods able to build flexible potential energy surfaces (see [18] and references therein).

Molecule-surface interactions have been usually described within the static surface Born-Oppenheimer approximation (SS-BOA). The validity of the SS approximation is strongly supported by the mass mismatch between the atoms of the molecule and the metal atoms of the surface whereas the BOA is supported by the velocity mismatch between nuclei and electrons, the latter ones being faster by few orders of magnitude than the former ones. Within the SS-BOA we describe the motion of the nuclei on a continuum potential energy surface (PES). Thus, the first step in any adiabatic simulation is to determine the electronic structure of the system, that is, the electronic landscape on which the nuclei move.

3.1. Potential Energy Surfaces. The first PES describing the electronic structure of a molecule-surface system was published in 1932 by Lennard-Jones [19]. This PES was built based on an analytical expression. Similar PESs based on London-Eyring-Polanyi-Sato (LEPS) potentials have been quite popular since then (see, e.g., [20-26]). Other analytical PESs are based on symmetry adapted functions $[27,28]$.

Although, for a number of molecule-surface systems, these analytical PESs have been able to describe successfully their electronic structure, the lack of flexibility of these PESs has impelled the development of methods based on interpolation of density functional theory (DFT) data. Generally speaking, the idea behind all these interpolation methods is quite similar. The first step is always to build a DFT data set; to this aim a number of high- and low-symmetry configurations (see Figure 4) are selected. For each configuration, defined by the position of the molecule over the surface ( $X$ and $Y$ ) and its orientation $(\theta$ and $\phi)$, a set of $r$ (atom-atom distance) and $Z$ (molecule-surface distance) values are computed-see Figure 5 for coordinates definition. In a second step the interpolation is performed over this data set.

First interpolation methods were applied to the development of low dimensional PESs [29, 30]. But, nowadays, to construct a PES based on the interpolation of a DFT data set, including all the molecular degrees of freedom (DOFs), has become a routine work thanks to the development of methods such as the corrugation reducing procedure (CRP) [31], the modified Shepard (MS) method [32,33], and the neural networks (NN) method [34].

The key idea behind the CRP method is that most of the corrugation of a molecule-surface PES is due to the atomsurface interactions and, therefore, the subtraction of this 


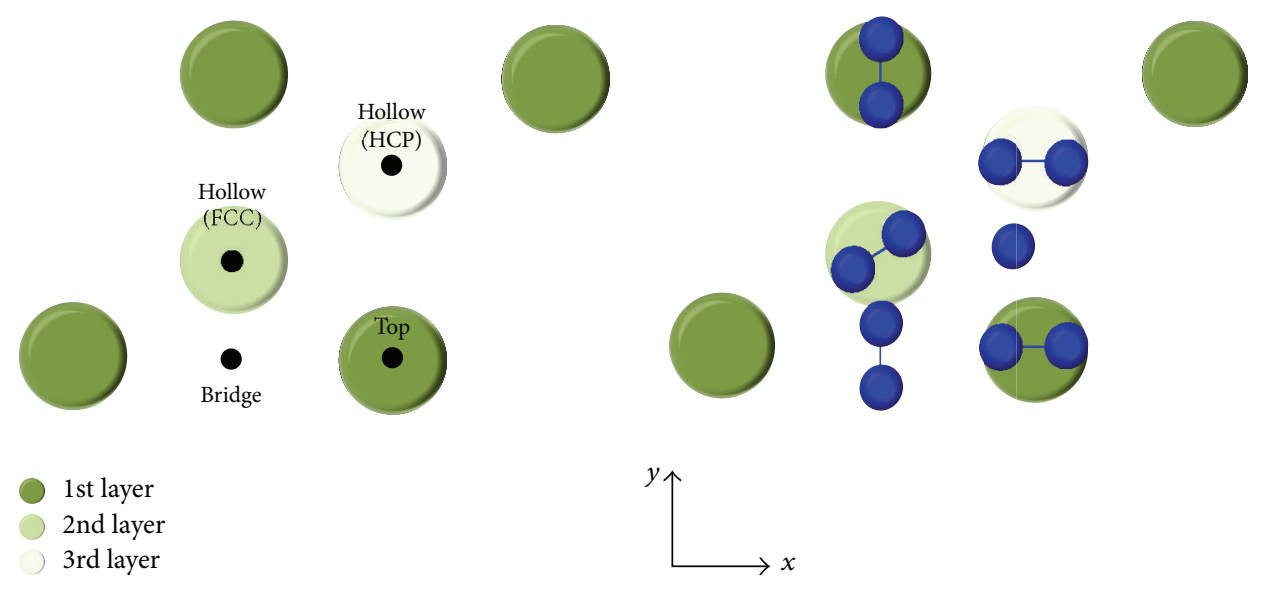

(a)

(b)

FIGURE 4: Schematic representation for a FCC(111) surface of (a) three high-symmetry surface sites; (b) some representative configurations of a diatomic molecule on the surface.

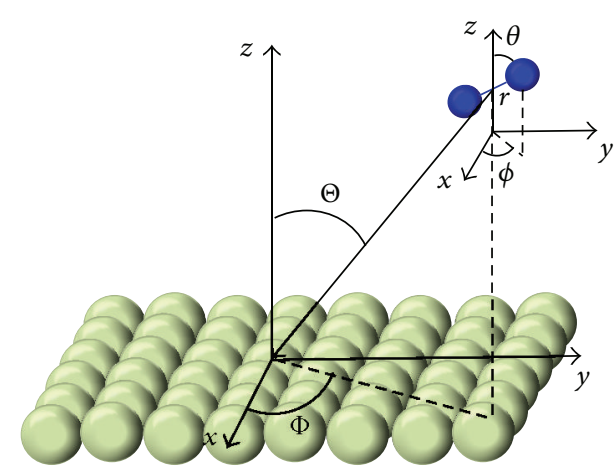

FIGURE 5: Schematic representation of the coordinate system.

atomic contribution from the PES leads to a much smoother function, which can be interpolated more accurately. Thus, the $6 \mathrm{D}$ PES can be written as

$$
V_{6 \mathrm{D}}^{\mathrm{CRP}}=S_{6 \mathrm{D}}(X, Y, Z, z, \theta, \varphi)+\sum_{i=1}^{2} R_{i}\left(X_{i}, Y_{i}, Z_{i}\right)
$$

where $S_{6 \mathrm{D}}$ is the smoother function (two-body term) and $R_{i}$ are the 3D PES representing the atom-surface interactions (one-body term). In the CRP method the interpolation is performed by combining analytical functions and numerical techniques. The major advantage of the CRP method is that the precision of the PES can be systematically improved by adding extra DFT data, whereas the major disadvantage is that it cannot be extended straightforwardly to describe polyatomic molecules (more than two atoms) interacting with surfaces. To date, the CRP method has been successfully used to build PESs for a wide variety of molecule-surface systems, for example $\mathrm{H}_{2} / \mathrm{Pd}(111)$ [31], $\mathrm{H}_{2} / \mathrm{Ni}(100)$, and $\mathrm{H}_{2} / \mathrm{Ni}(110)$ [35], $\mathrm{H}_{2} / \mathrm{Pt}(111)$ and $\mathrm{H}_{2} / \mathrm{Cu}(100)$ [36], $\mathrm{H}_{2} / \mathrm{Pd}(110)$ [37], $\mathrm{N}_{2} / \mathrm{W}(110)$ [38], $\mathrm{H}_{2} / \mathrm{NiAl}(110)$ [39], $\mathrm{H}_{2} / \mathrm{Pt}(211)$ [40], $\mathrm{H}_{2} / \mathrm{Ru}(0001)$ [41], $\mathrm{H}_{2} / \mathrm{Cu}(110)$ [42], $\mathrm{N}_{2} / \mathrm{W}(110)$ [43], $\mathrm{H}_{2} / \mathrm{W}(100)$, and $\mathrm{H}_{2} / \mathrm{W}(110)$ [44], $\mathrm{O}_{2} / \mathrm{Ag}(100)$ [45], $\mathrm{H}_{2} / \mathrm{Cu} / \mathrm{Ru}(0001)$, and $\mathrm{H}_{2} / \mathrm{Pd} / \mathrm{Ru}(0001)$

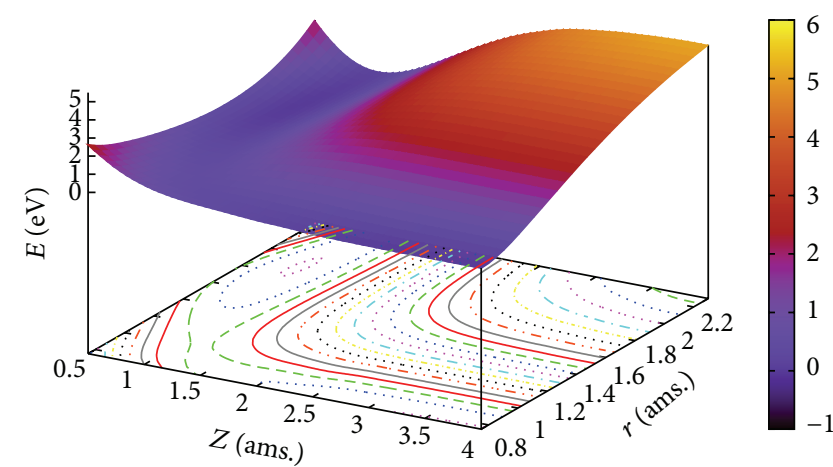

Figure 6: $2 \mathrm{D}$ cut through the $\mathrm{H}_{2} / \mathrm{Cu}(111)$ PES obtained by applying the CRP interpolation method to set of DFT-PW91 data. This 2D cut represents a $\mathrm{H}_{2}$ molecule dissociating on a bridge site (see Figure 4).

[46], $\mathrm{H}_{2} / \mathrm{Pd}(100)$ [47], $\mathrm{H}_{2} / \mathrm{Cu}(111)$ [48], and $\mathrm{H}_{2} / \mathrm{C}(1 \times 1)$ $\mathrm{Ti} / \mathrm{Al}(100)$ [49]. In Figure 6 we show, as an example, a typical $2 \mathrm{D}(r, Z)$ cut of the $6 \mathrm{D}$ PES obtained for the system $\mathrm{H}_{2} /$ $\mathrm{Cu}(111)$.

In the case of the MS method, initially developed to study gas phase reactions [50,51], the 6D PES is written as a weighted series of second-order Taylor expansions:

$$
V_{6 \mathrm{D}}^{\mathrm{MS}}=\sum_{j=1}^{N_{\mathrm{data}}} w_{j}\left(X_{i}\right) T_{j}\left(X_{i}\right),
$$

with $X_{i}$ representing the coordinates of the molecule, $w_{j}$ a normalized weigh function, and $T_{j}$ the second-order Taylor expansion of the potential centered on each data point. The main advantages of this method with respect to the CRP one are that (1) it can be extended straightforwardly to describe the interaction of polyatomic molecules with surfaces and (2) it requires a smaller number of $a b$ initio points, because it focuses the interpolation on the dynamically relevant regions of the PES, which are found by means of classical dynamics calculations. The MS method has been already used 
to describe the energy landscape of a number of systems, such as $\mathrm{H}_{2} / \mathrm{Pt}(111)$ [52], $\mathrm{N}_{2}$ /stepped-Ru(0001) [53], $\mathrm{N}_{2} / \mathrm{Ru}(0001)$ [54], $\mathrm{H}_{2} / \mathrm{CO} / \mathrm{Ru}(0001)$ [55], $\mathrm{H}_{2} / \mathrm{Pd}(111)$ [56], and $\mathrm{H} / \mathrm{H}-\mathrm{Si}(111)$ [57].

The NN method takes into account the symmetries underlying the system using nonfitting functions, which do not require any assumption about the functional form of the underlying problem. In this method the 6D PES is written as

$$
V_{6 \mathrm{D}}^{\mathrm{NN}}=f_{2}\left(W_{01}^{2}+\sum_{j} W_{j i}^{2} f_{1}\left(W_{o j}^{1}+\sum_{i=1}^{6} W_{i j}^{1} X_{i}\right)\right),
$$

where $X_{i}$ represent the six coordinates of the $\mathrm{H}_{2}$ molecule, $f_{2}$ and $f_{1}$ are nonlinear functions, and $W_{i j}$ are the parameters of the representation, the so-called weights. This method has been successfully applied to study, for example, the dissociative chemisorption of $\mathrm{H}_{2}$ on $\mathrm{K}(2 \times 2) / \mathrm{Pd}(100)$ [34], $\mathrm{O}_{2}$ on $\mathrm{Al}(111)$ [58], and $\mathrm{O}_{2}$ on $\mathrm{Ag}(111)$ [59]. Furthermore, this method can also be combined with the CRP method. For example, Ludwig and Vlachos [60] used a combination of these two methods to construct a PES representing the energy landscape for $\mathrm{H}_{2} / \mathrm{Pt}(111)$.

3.2. Dynamics. Within the BOA, once the electronic potential (the PES) is known, we can solve the time-dependent nuclear Schrödinger equation:

$$
\widehat{H} \Psi(\mathbf{R}, \mathbf{r} ; t)=i \frac{\partial \Psi(\mathbf{R}, \mathbf{r} ; t)}{\partial t} .
$$

In this equation, $\Psi(\mathbf{R}, \mathbf{r} ; t)$ represents the wave function describing the system, $\widehat{H}$ its Hamiltonian, and $\mathbf{R}$ and $\mathbf{r}$ represent the molecular center of mass and the molecular internal coordinates, respectively. In the case of a diatomic molecule, the Hamiltonian (in atomic units) can be written as

$$
\begin{aligned}
\widehat{H}= & -\frac{1}{2 M} \frac{\partial}{\partial Z^{2}}-\frac{1}{2 M \sin ^{2} \gamma}\left[\frac{\partial}{\partial X^{2}}-2 \cos \gamma \frac{\partial}{\partial X} \frac{\partial}{\partial Y}+\frac{\partial}{\partial Y^{2}}\right] \\
& -\frac{1}{2 \mu} \frac{\partial}{\partial r^{2}}+\frac{\hat{j}^{2}}{2 \mu r^{2}}+V_{6 \mathrm{D}}(X, Y, Z, r, \theta, \phi) .
\end{aligned}
$$

In this equation $\gamma$ is the angle between the $X$ and $Y$ coordinate axis [61] $\left(\gamma=90^{\circ}\right.$ if Cartesian coordinates are used). $M$ and $\mu$ are the total and reduced mass of the molecule, respectively; $\hat{j}$ is the rotational operator and $V_{6 \mathrm{D}}$ the PES.

There are several methods to solve the time-dependent Schrödinger (TDS) equation. In the time-dependent wave packet (TDWP) method as implemented by Kroes et al. [17, 62] the TDS is solved by numerically exact propagation of the wave packet using a time-independent basis-set. This method is divided into three steps:

(1) the choice of the initial wave packet;

(2) the propagation of the wave packet;

(3) the asymptotic analysis.
So that, at the end of the calculation, we obtain the monoenergetic state-resolved scattering probabilities $S(\nu, J$, $n, m$; $)$, with $v$ and $j$ being the vibrational and the rotational quantum numbers, $E$ the incidence energy of the molecule, and $(n, m)$ the diffraction state. From these $S(\nu, J$, $n, m$; $E$ ) probabilities, the monoenergetic dissociative adsorption probabilities $(S(\nu, J ; E))$ are computed as

$$
S(\nu, J ; E)=1-\sum_{n, m} S(\nu, J, n, m ; E)
$$

For further details on this method see $[3,17]$ and references therein.

A promising alternative method that can be also used to solve (5) is the so-called multiconfiguration time-dependent Hartree method (MCTDH) $[63,64]$. The main idea behind this method is to expand the wave function in a basis-set where not only the coefficient of the expansion, but also the basis functions themselves are time-dependent. The use of time-dependent basis-sets reduces their size, decreasing the computational cost with respect to the TDWP method.

Although, in general, the nuclear motion has to be described using quantum dynamics, classical dynamics is a very useful tool to get simple physical interpretations of quantum results and experimental measurements. In the classical dynamics method a classical trajectory is obtained by integration of the classical equations of motion.

We can integrate either the Hamilton equations of motion

$$
\begin{gathered}
\dot{q}_{i}=\frac{\partial H}{\partial p_{i}} \\
\dot{p}_{i}=-\frac{\partial H}{\partial q_{i}}
\end{gathered}
$$

$q_{i}$ and $p_{i}$ being the coordinates and the conjugated momenta of the system, respectively, or the Newton equations of motion

$$
M_{i} \frac{\partial^{2}}{\partial t^{2}} \mathbf{R}_{\mathbf{i}}=-\frac{\partial}{\partial \mathbf{R}_{\mathbf{i}}} V_{6 \mathrm{D}}\left(\mathbf{R}_{\mathbf{i}}\right)
$$

Disregarding the set of equations used, a classical dissociation probability is computed by averaging over internal coordinates and conjugated momenta of the molecules, which can be sampled using a standard Monte Carlo method.

In performing classical dynamics we can distinguish between pure classical and quasiclassical dynamics. In the latter, the zero point energy (ZPE) of the molecule is included in the calculation, whereas in the former the ZPE is assumed to be zero. In general, pure classical dynamics yields better results for nonactivated systems (see below for definition), and quasiclassical dynamics yields better results for activated systems (see below). For activated systems the ZPE may play a significant role due to the so-called vibrational softening, which happens whenever a molecule approaches an attractive surface. In this case, the attractive force between the atoms of the molecule and the surface becomes larger than the intramolecular force, and as a result the force constant associated with the vibrational motion is reduced, which induced 

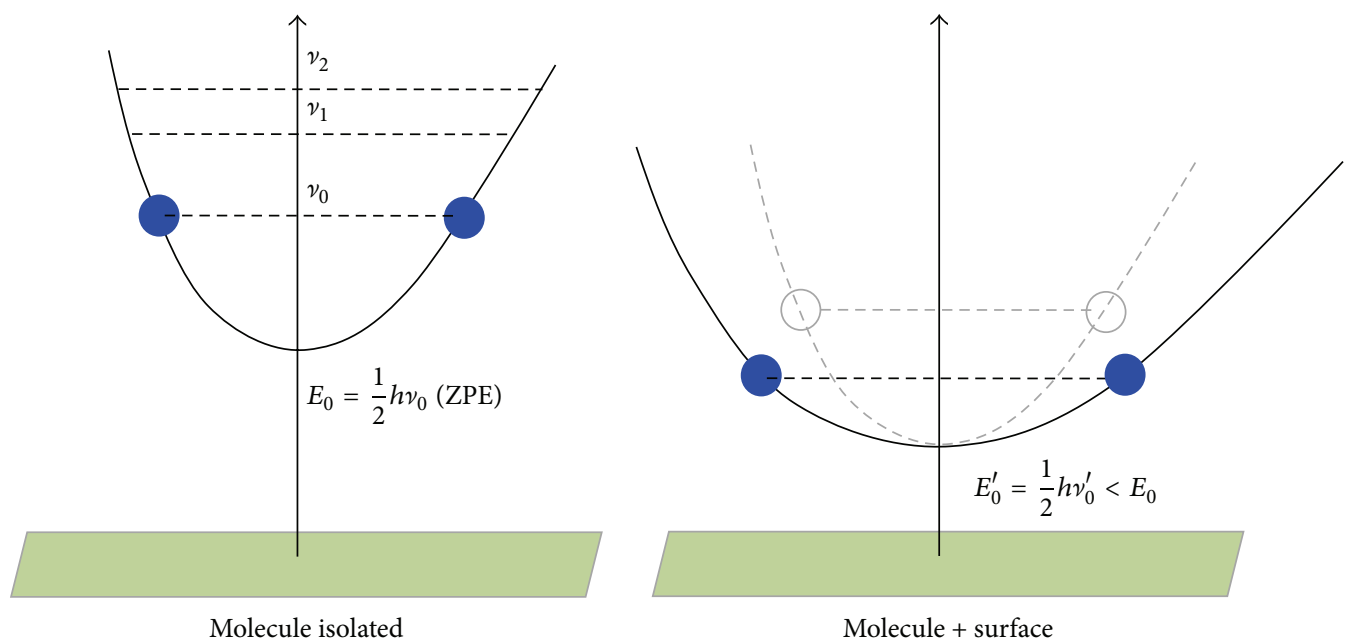

FIGURE 7: Schematic representation of the vibrational softening.

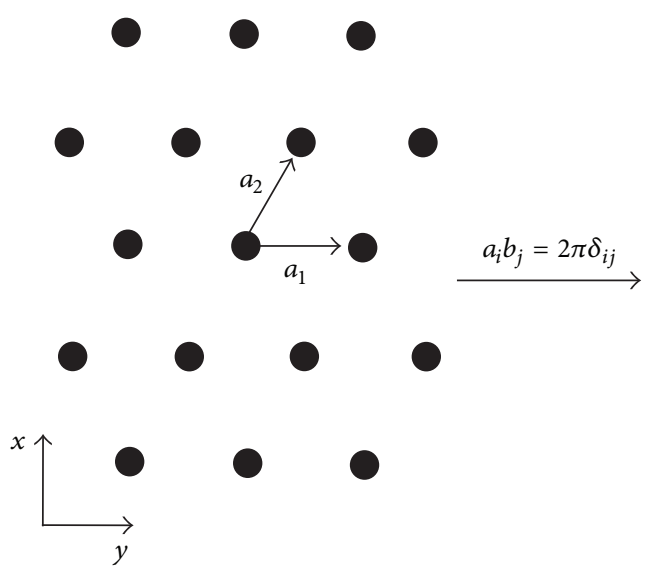

(a)

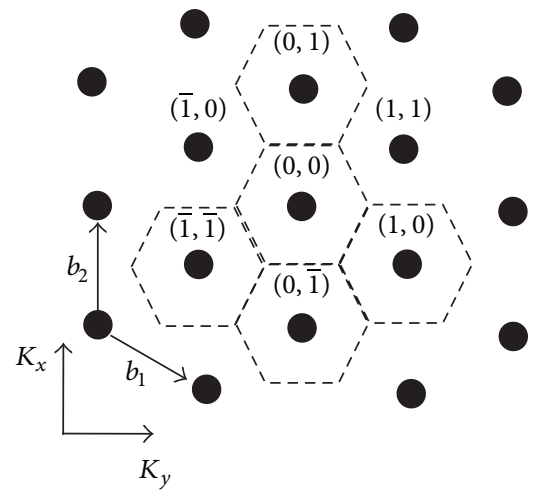

(b)

Figure 8: Real lattice (a) and reciprocal lattice (b) for a FCC surface. The numbers within parentheses indicate the diffraction peaks. The dashed hexagons show the Wigner-Seitz cells corresponding to some of the lattice points.

a decrease of the potential well curvature and, therefore, a decrease of the ZPE value (see Figure 7). This adiabatic energy transfer from vibration to translation is used by the molecule to overcome the reaction barrier and dissociate.

Classical dynamics can also be used to study quantum observables, such as rotational and vibrational quantum numbers $[65,66]$ and even diffraction probabilities [67-69]. Simulating rotational excitation using classical dynamics is possible by evaluating the closest integer that satisfied the quantum rigid rotor formula $\left[-1+\left(1+4 L^{2} / \hbar^{2}\right)^{(1 / 2)}\right] / 2, L$ being the classical angular momentum of the molecule. In the case of vibrational excitation, vibrational quantum numbers can be simulated by evaluating the closest integer that satisfied $S / \pi-1 / 2, S$ being the action variable. To simulate diffraction probabilities, the parallel momentum change is discretized by dividing the reciprocal space using as pattern the WignerSeitz cell associated with each lattice point $(n, m)$ (see Figure 8). Then, the classical diffraction probability, for a given diffraction peak $(n, m)$, is given by the number of trajectories leading to a parallel momentum change contained in the Wigner-Seitz cell of the $(n, m)$ peak, divided by the total number of trajectories.

\section{A Little of History: From Low to High Dimensional Simulations}

First quantum dynamics calculations of dissociative adsorption of a diatomic molecule on a metal surface were performed by Jackson and Metiu [70], for $\mathrm{H}_{2} / \mathrm{Ni}(100)$. In this study only the molecular bond $(r)$ and the distance moleculesurface $(Z)$ (see Figure 5) were taken into account; that is, only the vibrational and the translational motions towards the surface were properly described. Two years later a similar study was performed for $\mathrm{H}_{2} / \mathrm{Cu}(100)$ [71]. In 1992 Sheng and Zhang published the first 3-dimensional (3D) quantum study for $\mathrm{H}_{2} / \mathrm{Ni}(100)$ [72], including, in addition to $r$ and $Z$, the polar rotational motion $\theta$ (see Figure 5). Similar 3D studies were performed by Mowrey [73] for $\mathrm{H}_{2}\left(\mathrm{D}_{2}\right) / \mathrm{Ni}(100)$ 
and by Darling and Holloway [74] and Dai and Zhang [75] for dissociative adsorption of $\mathrm{H}_{2}$ on several $\mathrm{Cu}$ surfaces. Other 3D simulations were performed by taking into account $r, X$ (translational motion along the line joining two surface atoms neighbors-see Figure 5) [76]. More complex simulations including a forth degree of freedom were carried out since $1994[77,78]$. These simulations included $r, Z, \theta$, and $\phi$ $[24,79,80]$, with $\phi$ representing the molecular azimuthal motion (see Figure 5) or $r, Z, X$, and $Y-Y$ being the axis perpendicular to $X$ (see Figure 5).

Full dimensional quantum calculations, including the 6 DOFs of the molecule, were performed for $\mathrm{H}_{2} / \mathrm{Pd}(100)$ in 1995 [28] and later on for $\mathrm{H}_{2} / \mathrm{Cu}(111)$ [61] and $\mathrm{H}_{2} / \mathrm{Cu}(100)$ $[81,82]$. See Section 5 for more examples.

\section{Six-Dimensional Molecule-Surface Simulations}

When a light diatomic molecule approaches a metal surface, at low energy (up to few eV's), the molecule can either dissociate or get reflected. Relative to the behavior of the dissociative adsorption probability as a function of the molecular incidence energy, diatomic molecule-metal surface systems are classified as activated and nonactivated systems (see Figure 9). Activated systems show a monotonous increase of the dissociative adsorption probability as a function of the incidence energy. This behavior is due to the presence of a minimum reaction barrier (MRB) in PES. At this point, it should be pointed out that the MRB is only one of the characteristics of the PES that influence the interaction between a molecule and a surface, but it is not the only one, as it is shown in the following examples. If the PES does not present a MRB (although other barriers are present in the PES), the dissociative adsorption probability exhibits a nonmonotonous behavior. The dissociation probability first decreases when the incidence energy increases, reaching a minimum, and then it increases with the incidence energy. This nonmonotonous behavior is associated with the socalled dynamic trapping $[83,84]$. At low incidence energies the molecules are attracted by the attractive regions of the PES and get trapped on the surface, rebounding several times until they find a reactive path, that is, a path without a barrier, and dissociate. The trapping probability decreases when the incidence energy increases, decreasing the dissociation probability. On the contrary, the direct dissociation probability, which only depends on the dissociation barriers, increases monotonously with the incidence energy. The combination of both phenomena yields this characteristic nonmonotonous behavior (see Figure 10). In the following, we show few significant examples of both activated and nonactivated systems.

5.1. Dissociative Adsorption of $\mathrm{H}_{2}$ on Metal Surfaces. The dissociative adsorption probability of $\mathrm{H}_{2}$ on different metal surfaces has been widely studied during the last 20 years. This phenomenon is the first step in hydrogenation and dehydrogenation processes, which are of most importance for many industrial processes. From a purely theoretical point of view, the interest in these systems resides in the fact that

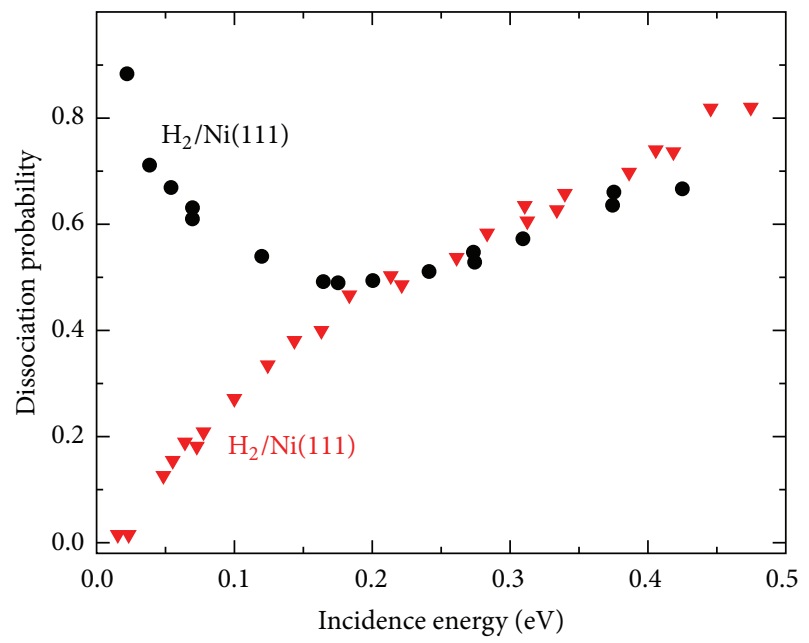

FIGURE 9: Dissociation probability of $\mathrm{H}_{2}$ on $\mathrm{Ni}(111)$ (black circles) and $\mathrm{Ni}(110)$ (red triangles). These systems are nonactivated and activated, respectively. Experimental data are taken from [176].

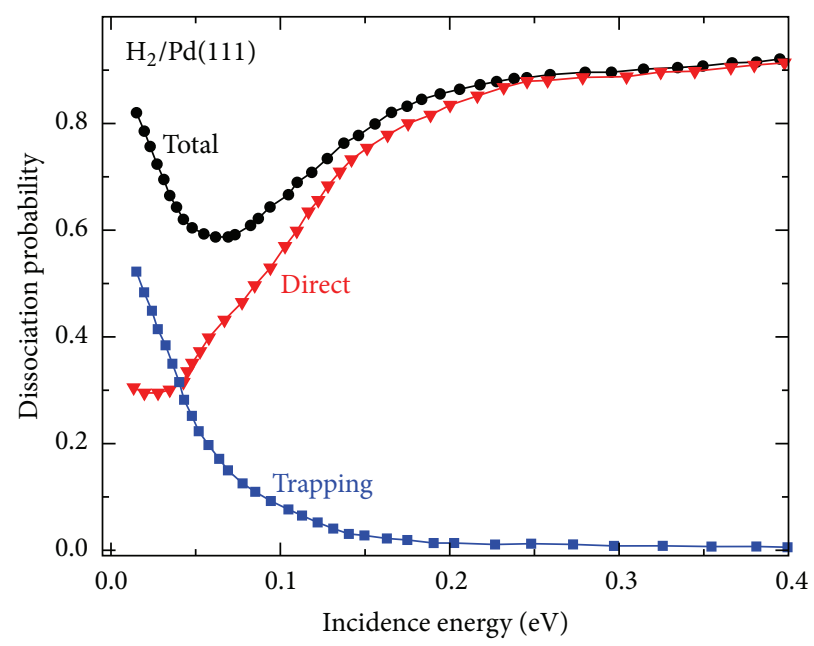

FIgURE 10: Theoretical dissociation probability versus incidence energy for $\mathrm{H}_{2} / \mathrm{Pd}(111)$. Black line with circles: total probability; red line with triangles: direct probability; blue line with squares: trapping probability.

they are the simplest molecule-surface systems and, therefore, theoretical models and hypothesis can be more easily tested on them.

Since the development of efficient methods to build $6 \mathrm{D}$ PES, the number of theoretical simulations performed to study the dissociative adsorption of $\mathrm{H}_{2}$ on metal surfaces, pure metal, alloys, and precovered-metal surfaces has experienced a significant increase. For example, in Figure 11 we show the dissociative adsorption probability of $\mathrm{H}_{2}$, in its rovibrational ground state $(v=0, J=0)$, for a number of activated surfaces-this is merely a sample of systems, by no means a complete list. From this figure two conclusions can be extracted: (i) although, qualitatively speaking, for all the systems the dissociative adsorption probability increases 


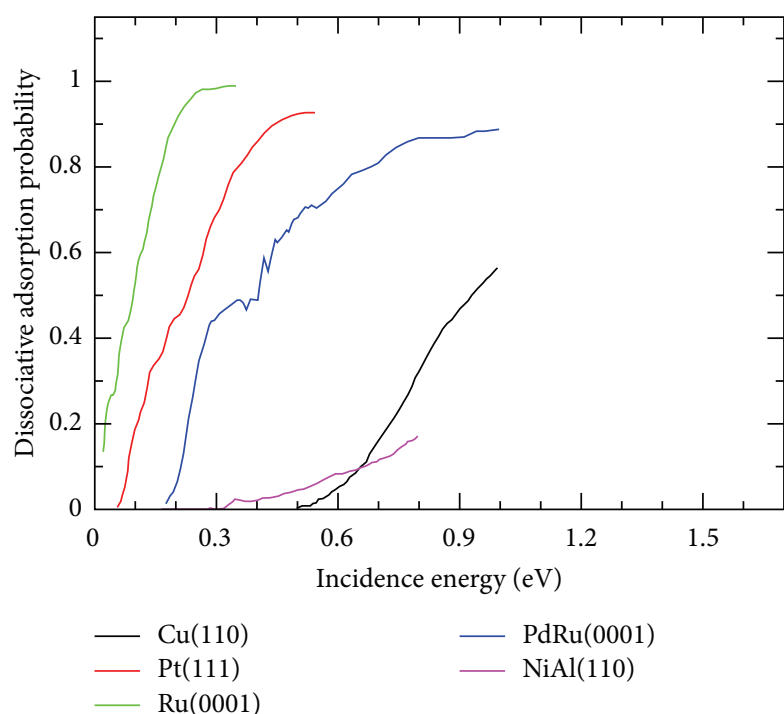

(a)

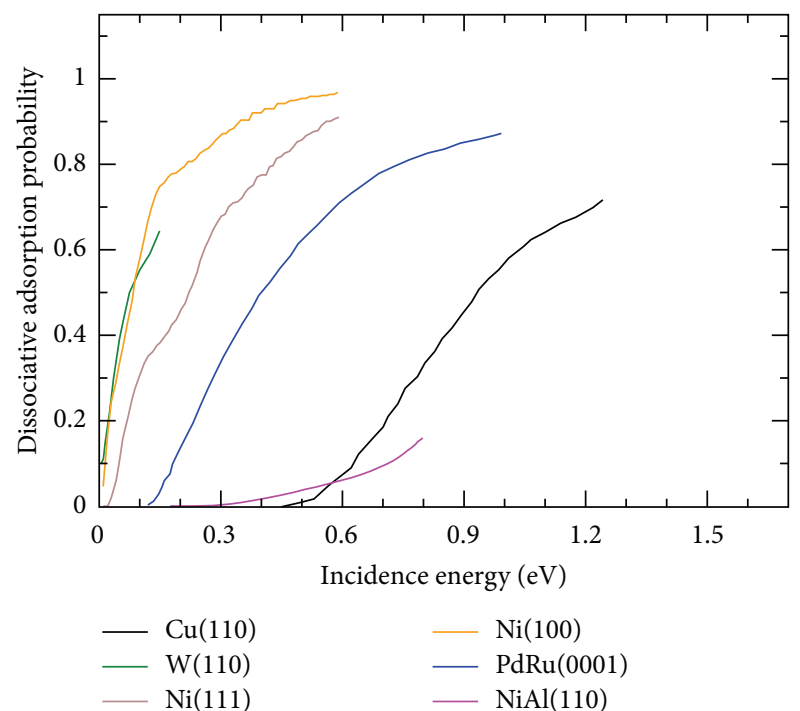

(b)

FIGURE 11: Dissociative adsorption probability of $\mathrm{H}_{2}$, in its rovibrational ground state $(v=0, J=0)$, as a function of the incidence energy. (a) Quantum calculations for $\mathrm{Cu}(110)$ (black line) [105]; Pt(111) (red line) [177]; Ru(0001) (green line) [41]; PdRu(0001) (blue line) [178]; NiAl(110) (magenta line) [179]. (b) Quasiclassical calculations for $\mathrm{Cu}(110)$ (black line) [42]; W(110) (green line) [44]; Ni(111) (brown line) [35]; Ni(100) (orange line) [35]; PdRu(0001) (blue line) [46]; NiAl(110) (magenta line) [39].

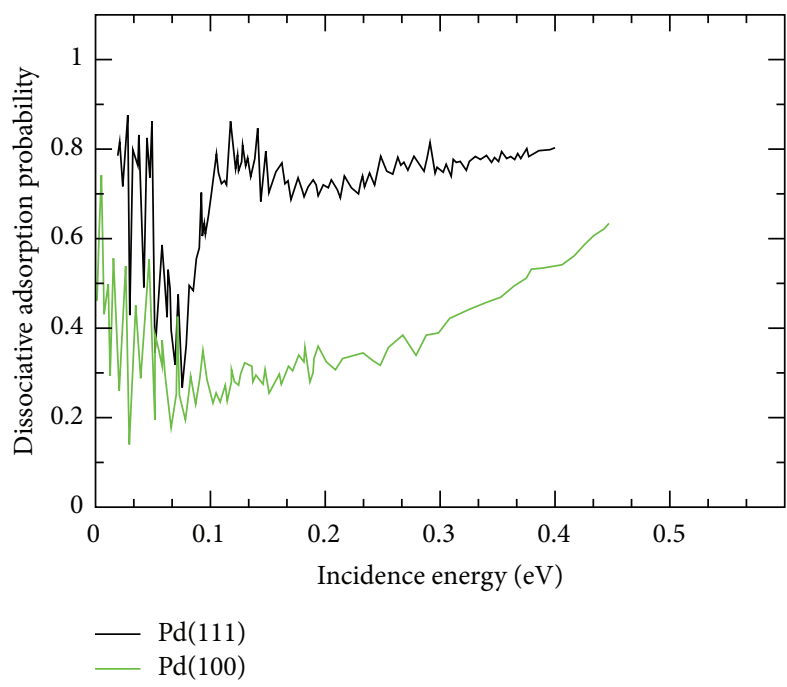

(a)

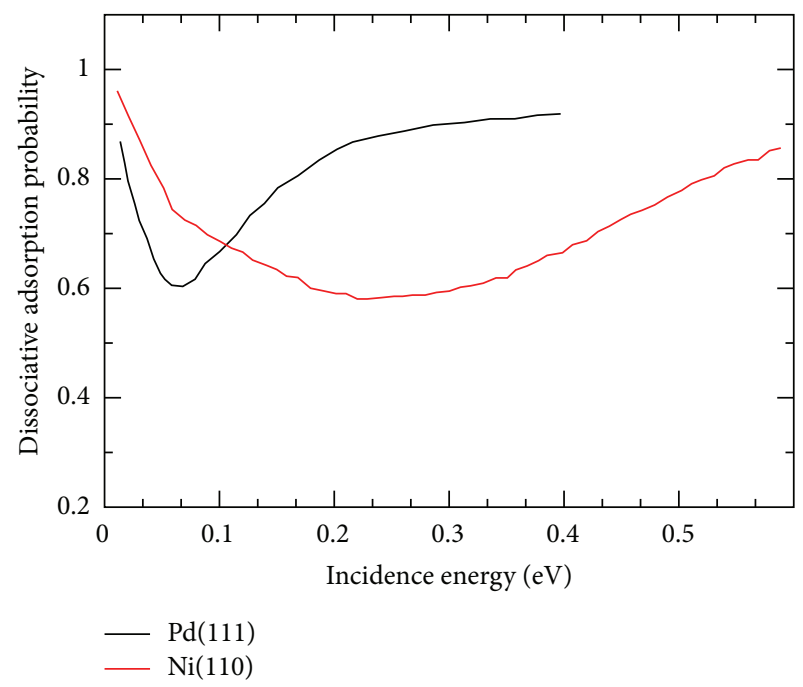

(b)

FIGURE 12: Dissociative adsorption probability of $\mathrm{H}_{2}$, in its rovibrational ground state $(v=0, J=0)$, as a function of the incidence energy. (a) Quantum calculations for Pd(111) (black line) [62]; Pd(100) (green line) [28]. (b) Classical calculations for Pd(111) (black line) [83]; Ni(110) (red line) [35].

monotonously when the incidence energy increases, the minimum energy leading to reaction and the slope of the curve vary from one system to another; (ii) by comparing quantum (Figure 11(a)) and classical (Figure 11(b)) calculations we can conclude that classical (quasiclassical, in fact) dynamics simulations give qualitative good results, with a smaller computational cost.

In Figure 12 we show some examples of dissociative adsorption of $\mathrm{H}_{2}(v=0, J=0)$ on nonactivated surfaces. Once again we can see that the subtle behaviour of the dissociative adsorption probability as a function of the incidence energy is surface-dependent and that the classical trajectory method gives pretty good results in comparison with the more sophisticate, and computational demanding time, quantum calculations.

Among the systems mentioned above, $\mathrm{H}_{2} / \mathrm{Cu}$ has been considered for long time the prototypical activated system, which explains the huge number of experimental [8598] and theoretical [21, 24, 42, 61, 65, 75, 78, 79, 99-113] works devoted to this system. As a result of this important 
theoretical effort, $\mathrm{H}_{2} / \mathrm{Cu}(111)$ has been the first system in which theoretical simulations have been able to reproduce experimental observables with chemical accuracy [111]. To achieve this aim, beyond using a well chosen DFT functional, an appropriate comparison with experimental measurements was needed. Experimentally, dissociative adsorption probabilities are measured using SMB techniques. To know the characteristics of this beam, that is, its molecular rovibrational distribution and its energy width, is crucial to perform a meaningful comparison between theory and experiment. Thus, in order to compare with experiments, the theoretical monoenergetic state-resolved probabilities have to be convoluted using the SMB parameters. This convolution is performed through the following steps:

(1) The monoenergetic state-resolved dissociation probabilities $S(\nu, J ; E)$, obtained from the simulations, are used to compute monoenergetic probabilities $S\left(T_{n}\right.$; $E$ ), which only depend on nozzle temperature and on the incidence energy as

$$
S\left(T_{n} ; E\right)=\sum_{\nu, J} F_{B}\left(\nu, J ; T_{n}\right) S(\nu, J ; E),
$$

with $F_{B}\left(\nu, J ; T_{n}\right)$ being the Boltzmann factor given by

$$
F_{B}\left(\nu, J ; T_{n}\right)=\frac{(2 J+1) e^{-\left(E_{v} / k T_{n}\right)} \times w(J) e^{-\left(E_{r} / 0.8 k T_{n}\right)}}{N},
$$

where $N$ is the normalization factor and $w(J)$ is the factor characterizing the nuclear spin statistics-for example, in the case of $\mathrm{H}_{2}\left(\mathrm{D}_{2}\right) w(J=0)=(1 / 4)(2 / 3)$ and $w(J=1)=(3 / 4)(1 / 3)$.

(2) The monoenergetic dissociation probabilities $S\left(T_{n} ; E\right)$ are used to simulate experimental dissociation probabilities $\left(S\left(T_{n}\right)\right)$ performing a convolution over the distribution of the molecular beam using the expression

$$
S\left(T_{n}\right)=\frac{\int_{v=0}^{v=\infty} f\left(v ; T_{n}\right) S\left(T_{n} ; E\right) d v}{\int_{v=0}^{v=\infty} f\left(v ; T_{n}\right)},
$$

where the flux weighted velocity distribution $f\left(v ; T_{n}\right)$ is given by

$$
f\left(v ; T_{n}\right) d v=C v^{3} e^{\left[-\left(v-v_{0}\right)^{2}\right] / \alpha^{2}} d v,
$$

and $C$ (a constant), $\alpha$ (the width of the velocity distribution), and $v_{0}$ (the stream velocity) define the experimental molecular beam.

The parameters describing the molecular beam $\left(\alpha, v_{0}\right)$ may vary from one experiment to another one, which explains to a large extent the differences on the dissociation probabilities as a function of the incidence energy found experimentally for some systems. For example, experimental results on dissociative adsorption probabilities for $\mathrm{H}_{2} / \mathrm{Cu}(111)$ measured by Rettner et al. [92] are significantly smaller than that obtained by Berger et al. [86] (see Figure 13). At this point, it should be pointed out that for long time there

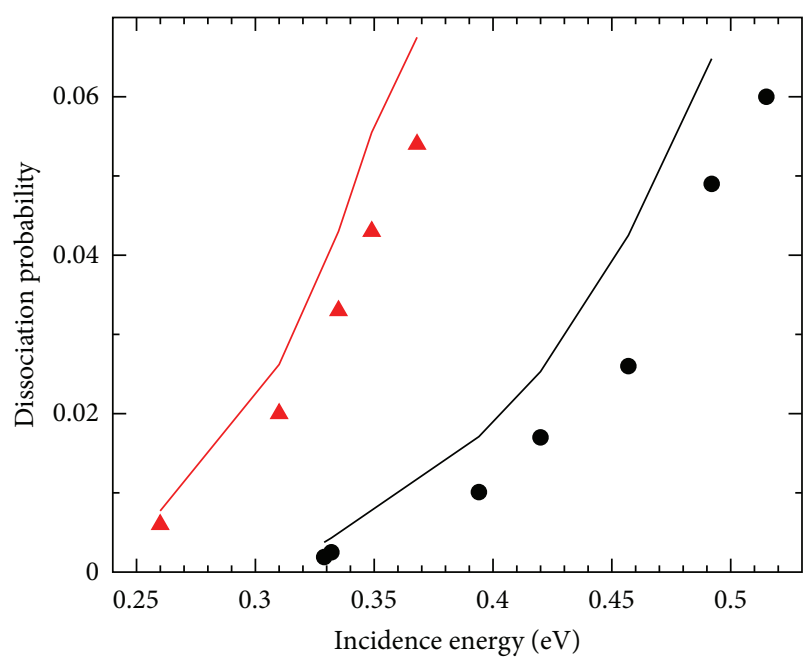

FIGURE 13: Dissociation probabilities as a function of the incidence energy for a pure $\mathrm{H}_{2}$ molecular beam on $\mathrm{Cu}(111)$. Solid lines: quantum theoretical simulations from [111]. Black circles: experimental data from [92]. Red triangles: experimental data from [86].

was not a clear explanation about why, at similar translational energies, these two sets of experimental dissociation probabilities differed that much. The explanation based on the different molecular beam parameters was offered in [111] through a detailed theoretical analysis. In [111] it was shown, on one hand, that an appropriate convolution of the theoretical probabilities, using the correct experimental molecular parameters, allows simulating both experimental data sets. And, on the other hand, an appropriate convolution of the theoretical probabilities is required in order to perform adequate analysis of the experimental data. This kind of convolution is, since then, performed routinely $[49,55]$.

These theoretical simulations can also be used to analyze the associative desorption [114] probability, and other observables [104], such as the following.

(i) the vibrational $\left(\chi_{v}\right)$ and rotational $\left(\chi_{J}\right)$ efficacy, which are used to evaluate the effectiveness of the vibrational and rotational energy in promoting reaction. $\chi_{v}$ can be computed as

$$
\chi_{v}=\frac{E_{0}(\nu=0, J=0)-E_{0}(v=1, J=0)}{E_{\nu}(v=1, J=0)-E_{\nu}(v=0, J=0)},
$$

where $E_{0}(v)$ is the incidence energy needed to obtain a reaction probability $S$ equal to half of the saturation value when the molecule is initially in the vibrational state $\nu$ and $E_{\nu}$ is the vibrational energy of the molecule in the gas phase. And $\chi_{J}$ can be computed by the slope of the line

$$
E_{0}(\nu=0, J)=C-\chi_{J} \times E_{J}(J)
$$

where $E_{J}$ represents the rotational energy. 
(ii) The associative desorption probabilities $\left(P_{D}(\nu, J ; E)\right)$ can be computed, invoking detailed balance, from the state-resolved dissociative adsorption probabilities as

$$
P_{D}(\nu, J ; E)=E e^{[-E / k T]} S(\nu, J ; E),
$$

where $T$ is the surface temperature and $k$ the Boltzmann constant.

(iii) The average desorption energies can be computed from state-resolved dissociative adsorption probabilities as

$$
\langle E(\nu, J)\rangle=\frac{\int S(\nu, J ; E) E^{2} e^{-E / k T} d E}{\int S(\nu, J ; E) E e^{-E / k T}} .
$$

(iv) The quadrupole alignment parameter $\left(A_{0}^{(2)}(J)\right)$ gives us a measurement of the reactivity of the molecule as function of its orientation, that is, as a function of $m_{j}$. $A_{0}^{(2)}(J)$ can be computed using the following equation:

$$
A_{0}^{(2)}(J)=\frac{\sum_{m_{j}=-J}^{J} S\left(\nu, J, m_{J} ; E\right)\left(3 m_{J}^{2}-J(J+1)\right) / J(J+1)}{\sum_{m_{j}=-J}^{J} S\left(\nu, J, m_{j} ; E\right)} .
$$

Thus, a positive value indicates a preference for reactivity when the molecule is oriented parallel (helicopter) to the surface $\left(m_{J}=J\right)$, whereas a negative value indicates, on the contrary, a preference for reactivity when the molecule is oriented perpendicular (cartwheel) to the surface $\left(m_{J}=0\right)$.

(v) The vibrational and rotational excitation probabilities of molecules scattered from surfaces.

5.2. Scattering of $\mathrm{H}_{2}$ from Metal Surfaces. The scattering of $\mathrm{H}_{2}$ from metal surfaces at low energy, below $1 \mathrm{eV}$, can be considered as the complementary process to dissociative adsorption-although, strictly speaking, it is the complementary process to sticking, which includes dissociative adsorption and molecular adsorption. By measuring the molecular distribution as a function of the scattering angle $(\Theta)$, and the molecular rotational and vibrational excitation upon scattering $[115,116]$, we can obtain extra information about the corrugation of the PES and, therefore, about the reactivity of the system. From a theoretical point of view, molecular scattering is a very useful phenomenon to test our tools. The behavior of a scattered molecule depends very much on the subtle characteristics of the PES; therefore the study of the molecular scattering procces allows us to evaluate the accuracy of PES and the accuracy of the dynamics methods.

For example, a detailed comparison between the experimental measurements and the theoretical 6D simulations, for vibrational and rotational survival probabilities and rotational excitation probabilities [65], had revealed the minor role played by nonadiabatic effects on the scattering of $\mathrm{H}_{2}$ and $\mathrm{D}_{2}$ from $\mathrm{Cu}(111)$ and accordingly on reactivity. On the other hand, TOF simulations for $\mathrm{H}_{2}(v=1, J=3) / \mathrm{Cu}(111)$ [117] have shown that the state-of-the-art adiabatic theoretical models

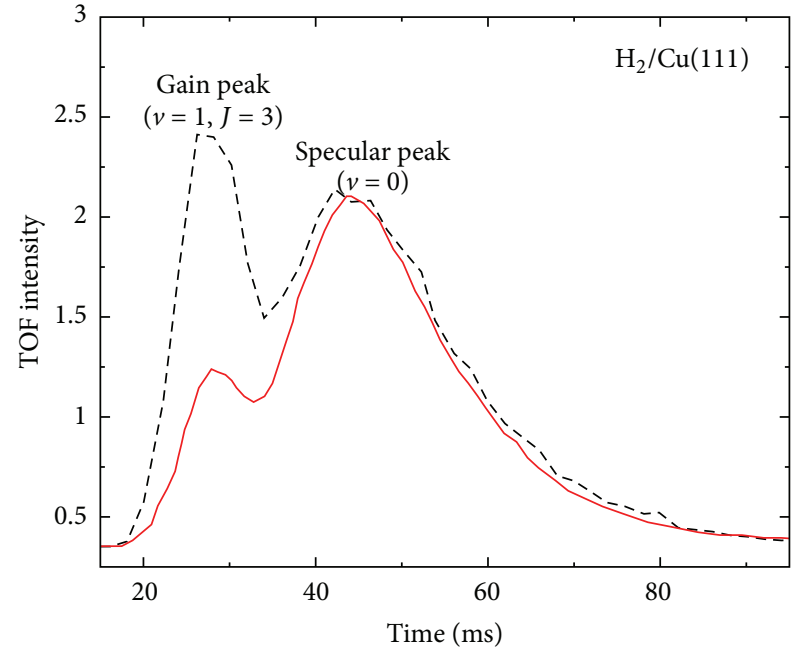

FIGURE 14: TOF spectrum of $\mathrm{H}_{2}(\nu=0)$ scattered from $\mathrm{Cu}(111)$. Black dashed line: experimental data $\left(T_{s}=400 \mathrm{k}\right)$ taken from [91]; red solid line: theoretical data from [117].

underestimate the vibrational excitation. This phenomenon can be observed in Figure 14, where it can be seen that the experimental gain peak (at short times), due to vibrational excitation from $\mathrm{H}_{2}(v=0)$ to $\mathrm{H}_{2}(v=1, J=3)$, is three times higher than the simulated one, whereas the agreement between theory and experiment for the specular peak is pretty good. In [117] it has been suggested that the discrepancy may be due to the use of the SS-BOA. As we discuss below (see Section 6), one the challenges that surface science theorists will have to face in the next future will be to go beyond this approximation.

The theoretical TOF probabilities shown in Figure 14 were computed by using the following equation:

$$
\begin{aligned}
f\left(t, T_{n}\right) & \\
=c+N \times & {\left[\left(\frac{v_{i}}{v_{0}}\right)^{4} \times\left[-\left(\frac{v_{i}-v_{0}}{\alpha}\right)^{2}\right]\right.} \\
& \times P\left(v=v^{\prime}, J=J^{\prime} \longrightarrow v^{\prime}, J^{\prime}\right) \\
+x_{t} \times( & \sum_{v J, v^{\prime} J^{\prime} \neq}\left(\frac{v_{i}^{3}}{v_{s} v_{0}^{4}}\right) \\
& \times\left(\frac{1}{x_{i} v_{i}^{-2}+x_{s} v_{i} v_{s}^{-3}}\right) \\
& \times \exp \left[-\left(\frac{v_{i}-v_{0}}{\alpha}\right)^{2}\right] \\
& \left.\left.\times w_{v J} \times P\left(v, J \longrightarrow v^{\prime}, J^{\prime}\right)\right)\right],
\end{aligned}
$$




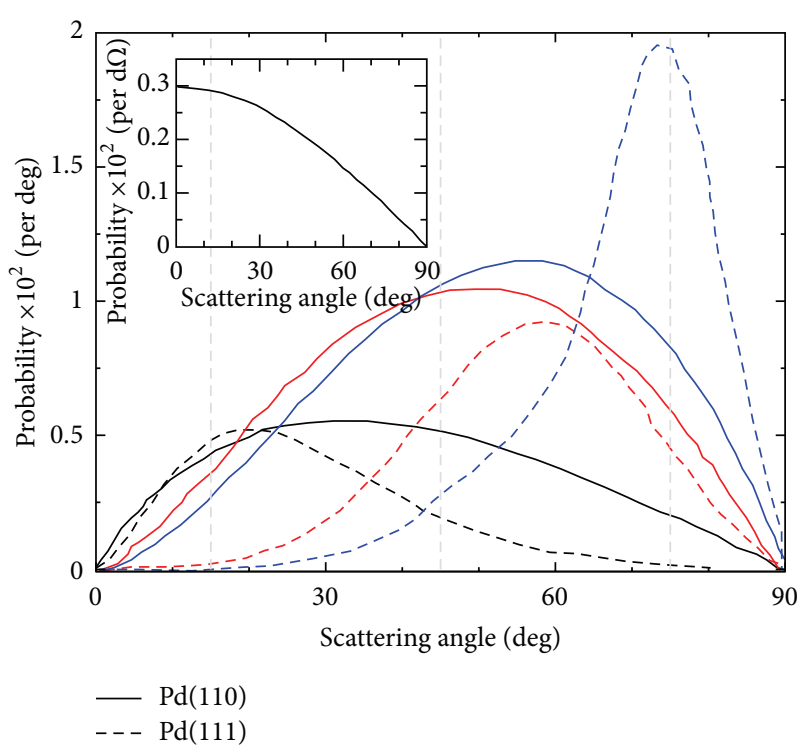

FIGURE 15: Reflection probabilities per degree as a function of the scattering angle $(\Theta)$ for $\mathrm{H}_{2} / \mathrm{Pd}(110)$ (solid lines) and $\mathrm{H}_{2} / \mathrm{Pd}(111$ ) (dashed lines). Data are taken from [118, 119], respectively. Black lines: incidence angle, $\Theta_{i}=15^{\circ}$; red lines: $\Theta_{i}=45^{\circ}$; blue lines: $\Theta_{i}=75^{\circ}$. Inset: reflection probability per solid angle as a function of the scattering angle for $\mathrm{H}_{2} / \mathrm{Pd}(110)\left(\Theta_{i}=45^{\circ}\right)$.

where $x_{i}\left(x_{s}\right)$ represents the distance travelled by the molecule from the source (surface) to the surface (detector), $v_{s}$ is the velocity of the scattered molecule, $w_{\nu, J}$ represents the Boltzmann population of the initial molecule state $(\nu, J)$ in the molecular beam divided by the population of the final state $\left(v^{\prime}, J^{\prime}\right)$ (at the nozzle temperature used in the experiment), and $P\left(v, J \rightarrow v^{\prime}, J^{\prime}\right)$ is the transition probability from the initial state $(\nu, J)$ to the final state $\left(v^{\prime}, J^{\prime}\right)$.

The angular distributions of $\mathrm{H}_{2}$, upon scattering from metal surfaces, have been studied, for example, for $\mathrm{Pd}(111)$ [118] and $\operatorname{Pd}(110)$ [119]. These studies revealed a quite different behavior of the angular distributions (see Figure 15). A detailed analysis of these distributions revealed that the $\mathrm{H}_{2}$ molecules are reflected closer to $\mathrm{Pd}(110)$ than to the $\operatorname{Pd}(111)$, and therefore they are more sensitive to the surface corrugation in the case of $\mathrm{Pd}(100)$. This analysis also allowed establishing the signature of the dynamic trapping, a cosinelike angular distribution of the reflection probability per solid angle (see inset Figure 15). Here, it is important to remark that the same cosine-like distribution is observed in trappingdesorption experiments [120]. But the origin of trappingdesorption is totally different from the origin of dynamic trapping. The former phenomenon is due to the thermal equilibrium, that is, to the energy exchange between the surface and the molecule, which requires long interaction times (millisecond) in contrast with dynamic trapping that is a faster process (picoseconds).

5.3. Diffraction of $\mathrm{H}_{2}$ from Metal Surfaces. The diffraction of hydrogen molecules from a metal surface [6] can be considered as a unique technique to gauge the moleculesurface PES and the dynamics. First detailed comparison between experimental diffraction data and 6D adiabatic quantum calculations was performed on $\mathrm{H}_{2} / \mathrm{Pt}(111)$ [121]. The agreement between the experimental diffraction peaks probabilities and the theoretical ones, for several incidence energies, was found to be remarkable-experimental results were extrapolated to $0 \mathrm{~K}$ surface temperature using the Debey-Wallet attenuation model (see [122] and references therein). This excellent agreement, together with the very good agreement obtained for dissociative adsorption [62], allowed discarding any significant role of nonadiabatic effects on this system. A good agreement with experimental diffraction peaks was also obtained for $\mathrm{D}_{2} / \mathrm{NiAl}(110)[123,124]$. Although, in this latter case, the agreement is not that good for rotationally inelastic diffraction (RID). In this case, it has been suggested that these discrepancies may be due to inaccuracies in the PES [124], discarding surface phonons and significant nonadiabatic effects.

The systems mentioned above are activated systems, i.e., under experimental conditions most of the molecules (if not all of them) are reflected, which simplify the uptake of experimental measurements. But diffraction of nonactivated systems represents a major challenge, because under experimental conditions most of the molecules dissociate; therefore, the number of the scattered molecules is very low, and the surface gets dirty (covered with hydrogen) very fast. Thus, the surface temperature needs to be kept high enough to avoid hydrogen coverage, increasing phonons background and making more difficult the extrapolation of the measurements to $0 \mathrm{~K}$. First measurements and subsequent simulations on these kinds of systems were carried out on $\mathrm{H}_{2} / \mathrm{Pd}(111)$ [123]. The most remarkable result obtained from this study was a pronounced out-of-plane diffraction, showing a highly corrugated PES. This pronounced out-of-plane diffraction was considered to be associated with high reactive systems. This study revealed the importance of scanning the whole space (in-plane and out-of-plane) in order to infer trustworthy properties of the PES. Diffraction was also studied for $\operatorname{Pd}(110)$ [125]. In this case, the theoretical simulations showed a large number of diffraction peaks, with very low probability, which prevent the experimental observation. The presence of many diffraction peaks with low intensity was considered a signature of the dynamic trapping.

On the other hand, diffraction measurements of $\mathrm{H}_{2}$ from $\mathrm{Cu}(111)$ have revealed a very low out-of-plane diffraction probability [69]. This result, together with previous results for $\mathrm{H}_{2}$ diffraction from $\mathrm{Pt}(111), \mathrm{NiAl}(110), \operatorname{Pd}(111)$, and $\mathrm{Pd}(110)$, was induced to suggest that the presence of intense out-ofplane diffraction peaks in the diffraction spectrum could be considered a signature of a notable dissociative adsorption probability. However, detailed analyses of the systems $\mathrm{H}_{2} / \mathrm{Ru}(0001), \mathrm{H}_{2} / \mathrm{Cu}(111)$, and $\mathrm{H}_{2} / \mathrm{CuRu}(0001)$ have shown that there is not a lineal relationship between dissociative adsorption and out-of-plane diffraction [69]. In Figure 16 we can observe that $\mathrm{H}_{2} / \mathrm{Ru}(0001)$ is the most reactive system, whereas the most intense out-of-plane diffraction is found for $\mathrm{H}_{2} / \mathrm{CuRu}(0001)$. 


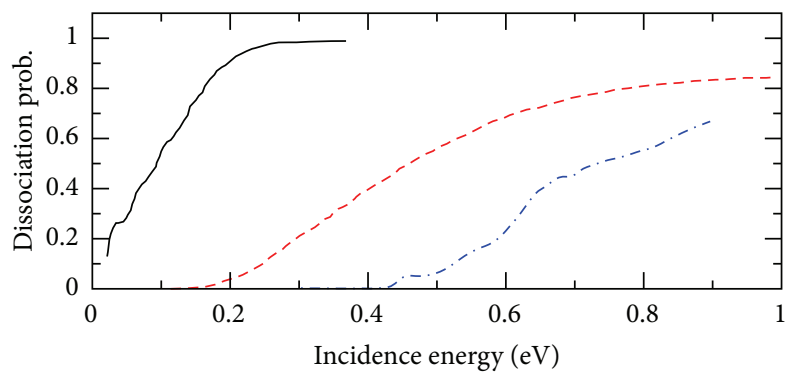

(a)

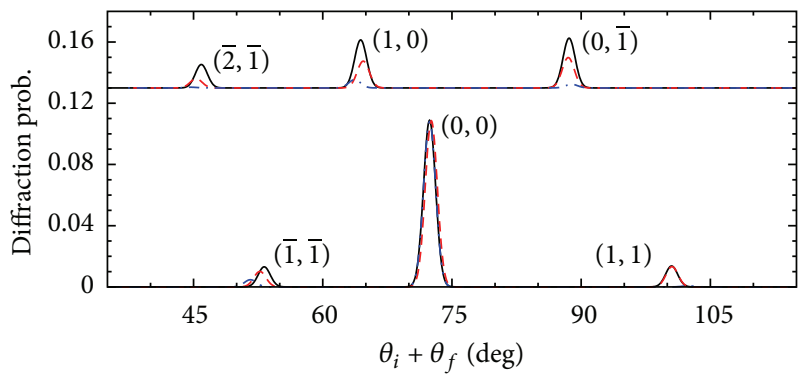

(b)

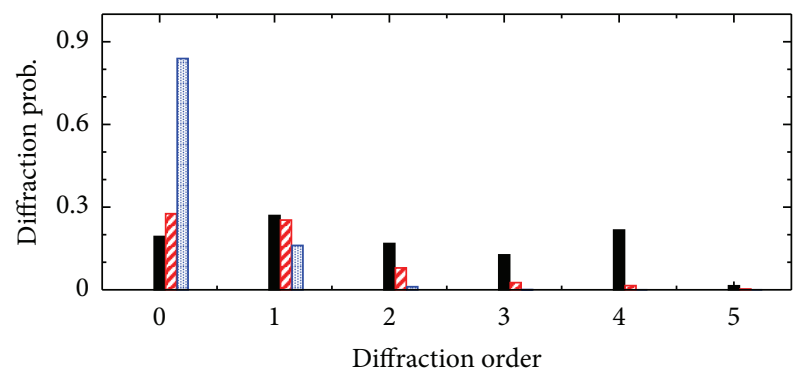

(c)

FIGURE 16: (a) dissociative adsorption probability of $\mathrm{H}_{2}$ as a function of the incidence energy. (b) in-plane and out-of-plane (see Figure 8) diffraction spectra for an incidence energy $=0.075 \mathrm{eV}$ and $\Theta_{i}=30^{\circ}$. Raw data have been convoluted using a Gaussian function of width $\sigma=0.7^{\circ}$, and the peaks have been normalized to the specular peak for $\mathrm{H}_{2} / \mathrm{CuRu}(0001)$. (c) diffraction probabilities as a function of the diffraction order-defined by concentric hexagons built around the specular peak; peaks lying on the same hexagon belong to the same order (see Figure 8). Solid black bars: $\mathrm{CuRu}(0001)$; dashed red bar: $\mathrm{Ru}(0001)$; dotted bars: $\mathrm{Cu}(111)$.

\section{Perspectives and Future Challenges}

The surface static Born-Oppenheimer approximation has been essential to advance in the field of surface dynamics, but recent experiments on reactive and nonreactive scattering have questioned its applicability [126-128]. Therefore, some of the major challenges that theorists are facing in the next future are related to the development of theoretical models, which will allow including accurately electrons and phonons excitations $[129,130]$.

The need for such models is becoming more and more visible as surface scientists are facing more and more complex systems, for example, heavy and polyatomic molecules interacting with surfaces, such as $\mathrm{NO}[128,131,132], \mathrm{O}_{2}[45$, $58,59,133], \mathrm{N}_{2}[38,43,134-136]$, and $\mathrm{CH}_{4}$ [137-143].

6.1. Beyond the Born-Oppenheimer Approximation. The possible influence of nonadiabatic effects and concretely of electron-hole (e-h) pair excitations is currently under debate within the surface dynamics community. This debate comes from experiments showing unexpected results. For example, experimental results for $\mathrm{N}_{2} / \mathrm{Ru}(0001)$ showing very low dissociative adsorption probabilities, for incidence energies well above the minimum reaction barrier $[144,145]$, or the isotope-dependence behavior found for vibrational deexcitation of $\mathrm{H}_{2}$ and $\mathrm{D}_{2}$ upon scattering from $\mathrm{Cu}(100)$ have been considered as a fingerprint of strong nonadiabatic effects $[115,116]$. This conclusion was supported by low dimensional adiabatic calculations, which were unable to reproduce these experimental results $[145,146]$. However, subsequent theoretical studies, using high (6D) dimensional adiabatic calculations, have refuted this conclusion [54, 65]. But stronger experimental indications of nonadiabatic effects, coming from experiments showing e-h pair excitation accompanying molecular chemisorption [126] and ejection of electrons accompanying scattering of highly vibrationally excited molecules, call for the development of theoretical methods able to account for these effects [128].

The first attempts to include electron-hole pair excitations in dynamics studies of molecular processes on metal surfaces date back to the 80s [147-149]. Most recently, several DFTbased approximate methods have been proposed to include nonadiabatic effects in the dynamics. Luntz et al. [146] have proposed to compute friction coefficients $(\eta)$, describing electron-hole pair damping along the minimum energy barrier reaction path. This method allows performing quasiclassical nonadiabatic dynamics simulations on 2D PESs. But it cannot be extrapolated straightforwardly to multidimensional (6D) calculations. In view of the importance of taking into account the full dimensionality the system may have on the appropriate description of many molecule-surface phenomena, the applicability of this method is rather limited. Juaristi et al. [150] have proposed an approximate method to include nonadiabatic effects by keeping the multidimensionality of the problem. The main approximation of this method, from now on called LDFA (local density friction approximation), is to consider that the atoms of the molecule move independently. Using the LDFA, the nonadiabaticity is introduced in the classical equations of motion, for each atom of the molecule, through a dissipative force. Thus, the equation of motion for each atom can be written as

$$
m_{i} \frac{d^{2} r_{i}}{d t^{2}}=-\nabla_{i} V_{6 \mathrm{D}}\left(r_{i}, r_{j}\right)-\eta\left(r_{i}\right) \frac{d r_{i}}{d t},
$$

where the second term in the right-hand side of this equation is the dissipative force experienced by each atom.

Shenvi et al. [151] have proposed to take into account nonadiabatic effects by using an independent electron surface hopping (IESM), in which the energy transfer to e-h pair excitations is described by considering hops between electronic adiabatic states. Using the IESM model, these authors have 


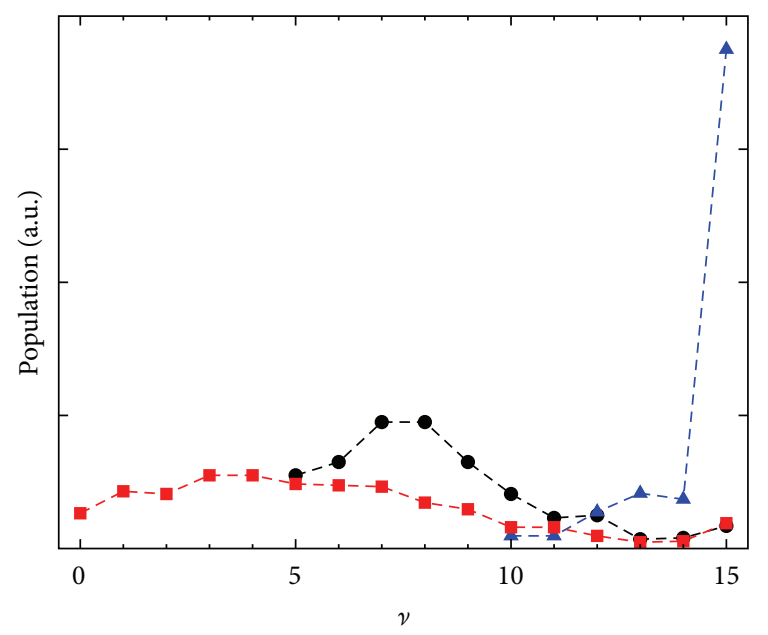

๑- Exp

- A Adiabatic

- - IESH

Figure 17: Vibrational state population distribution of $\mathrm{NO}(\nu=$ $15) / \mathrm{Au}(111)$ as a function of the vibrational quantum number $(v)$. Black circles: experimental results from [131]; blue triangles: adiabatic theoretical results from [132]; red squares: IESM results from [132].

been able to reproduce qualitatively the experimental vibrational deexcitation probabilities obtained for $\mathrm{NO} / \mathrm{Au}(111)$ $[131,132]$ (see Figure 17), a system for which nonadiabatic effects have been found to be quite important $[128,152]$. However, a model to accurately describe nonadiabatic effects in reactive and nonreactive scattering of molecules from metal surface is still to be developed.

6.2. Surface Temperature: The Effect of Phonons. Although the static surface approximation has yielded qualitatively good results for reactive and non-reactive scattering for many molecule-surface systems, including the effect of surface temperature may be crucial to accurately describe some systems and/or observables. For example, there are strong indications that vibrational excitation of $\mathrm{H}_{2}$ upon scattering from $\mathrm{Cu}(111)$ can only be accurately described if phonons are taken into account [117].

So far, several methods have been proposed to include surface temperature, all of them within the classical dynamics framework. The simple one is the so-called surface oscillator (SO) model [153], in which a 3D harmonic oscillator is used to describe the collective motion of the surface atoms (see Figure 18). In this model, the coupling between the molecule and the surface atoms motion is described by a rigid coordinate shift of the $6 \mathrm{D}$ PES. Thus, the 9D PES can be written as

$$
\begin{aligned}
V_{9 \mathrm{D}}\left(R_{A}, R_{B}, R_{S}\right)= & V_{6 \mathrm{D}}\left(R_{A}-R_{S}, R_{B}-R_{S}\right) \\
& +\frac{M}{2}\left(w_{x}^{2} X_{S}^{2}+w_{Y}^{2} Y_{S}^{2}+w_{Z}^{2} Z_{S}^{2}\right),
\end{aligned}
$$

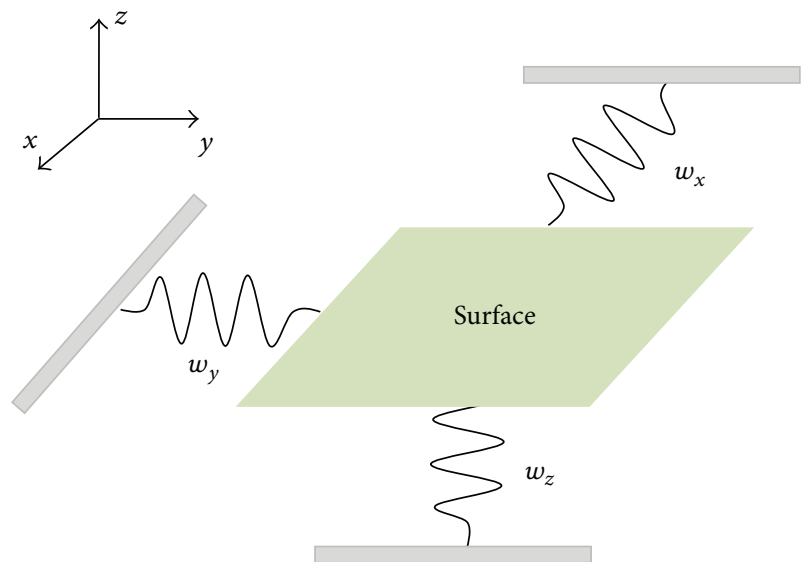

FIGURE 18: Schematic representation of the surface oscillator model.

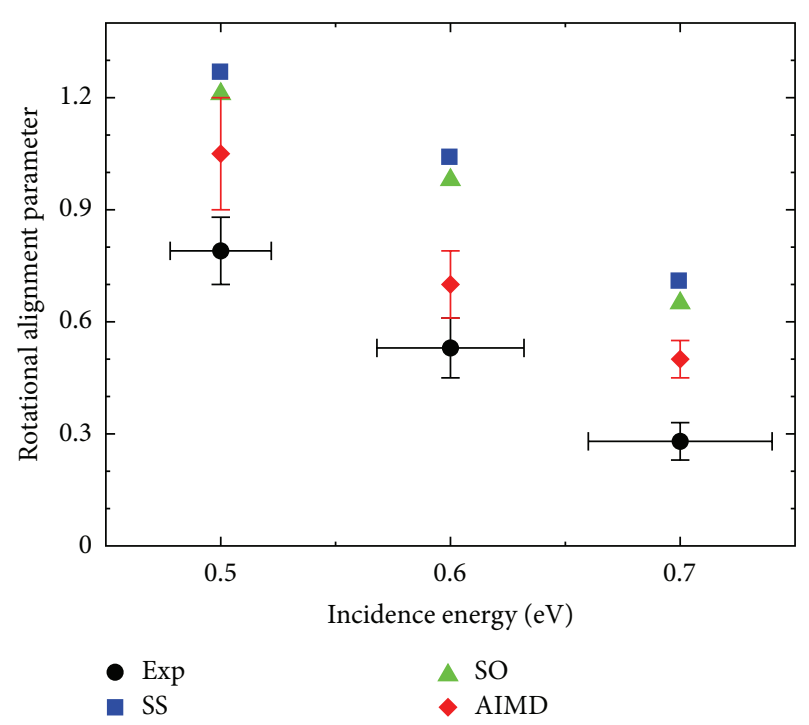

FIGURE 19: Quadrupole alignment parameter as a function of the incidence energy for $\mathrm{H}_{2}$ on $\mathrm{Cu}(111)$. Black solid circles: experimental results from [93]; blue squares: static surface approximation results from [104]; green triangles: surface oscillator model results from [104]; red diamonds: AIMD results from [106].

where $w_{X, Y, Z}$ are the surface oscillation frequencies, $M$ is the surface atoms mass, and $R_{A, B, S}$ are the vectors $(X, Y, Z)$ defining the position of the molecule and surface atoms.

This method has been successfully used to study, for instance, the rotational excitation of $\mathrm{H}_{2}$ upon scattering from $\operatorname{Pd}(111)$ [66] and the quadrupole alignment parameters for $\mathrm{D}_{2} / \mathrm{Cu}(111)$. In the latter case, it was found that though the SO model yields better results than the static surface one, it is still too simple to obtain chemical accuracy [104]. Much better results have been obtained using ab initio molecular dynamics (AIMD) [106] (see Figure 19). This method allows the motion of the surface atoms in such a way that the intricate molecule-phonons coupling can be taken into account. AIMD, used for the first time to study moleculesurface reactions by Groß and Dianat [154], computes the forces on the fly which increases hugely the computational 


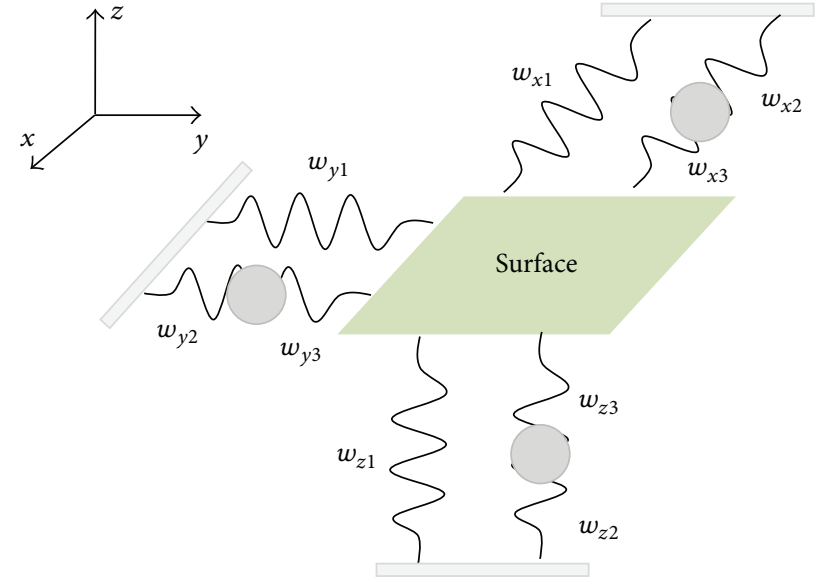

FIgURE 20: Schematic representation of the generalized Langevin oscillator model.

cost, limiting the number of classical trajectories that can be computed.

An improvement over the SO model is the generalized Langevin oscillator (GLO) model [155] adapted to study molecule-surface interactions by Busnengo et al. [156] to analyze the effect of surface temperature in the dynamic trapping mediated adsorption process of $\mathrm{H}_{2}$ on several Pd surfaces. This method includes dissipation and thermal fluctuations, thanks to ghost 3D oscillator (see Figure 20), which is coupled to the surface oscillator. In this model, the ghost oscillator is subject to friction and random forces. Within this framework model, the equations of motion for the 12 coordinates of the system are given by

$$
\begin{gathered}
\frac{d^{2} R_{A, B}}{d t^{2}}=-\frac{1}{m_{A, B}} \nabla_{R_{A, B}} V_{6 \mathrm{D}}\left(R_{A}-R_{S}, R_{B}-R_{s}\right), \\
\frac{d^{2} R_{s}}{d t^{2}}=-\frac{1}{m_{s}} \nabla_{R_{S}} V_{6 \mathrm{D}}\left(R_{A}-R_{s}, R_{B}-R_{S}\right)-\omega_{S}^{2} R_{S}+\Lambda_{g s} U, \\
\frac{d^{2} U}{d t^{2}}=-\omega_{g}^{2} U+\Lambda_{g s} R_{S}+\gamma_{g} \frac{d U}{d t}+F_{r}(\Delta t) .
\end{gathered}
$$

Here $U\left(U_{x}, U_{y}, U_{z}\right)$ and $\omega_{g}$ are the coordinates and frequency matrix associated with the ghost oscillator, $\Lambda_{g s}$ is the coupling matrix, which couples the ghost and the surface oscillators, $\gamma_{g}$ represents the damping matrix associated with the friction force, and $F_{r}(\Delta t)$ is the random force.

GLO model has been used by Goikoetxea et al. [135] to study the molecular adsorption of $\mathrm{N}_{2}$ on $\mathrm{Fe}(110)$, showing a strong dependence of the maximum of the molecular adsorption on the surface temperature (see Figure 21). This method has been also used to study, for example, the scattering of $\mathrm{N}_{2}$ from W(110) [136].

Some effort to include surface phonons effects in quantum dynamics has been recently done [157]. In this case, a $7 \mathrm{D}$ model including the perpendicular motion of the second layer atom was proposed.

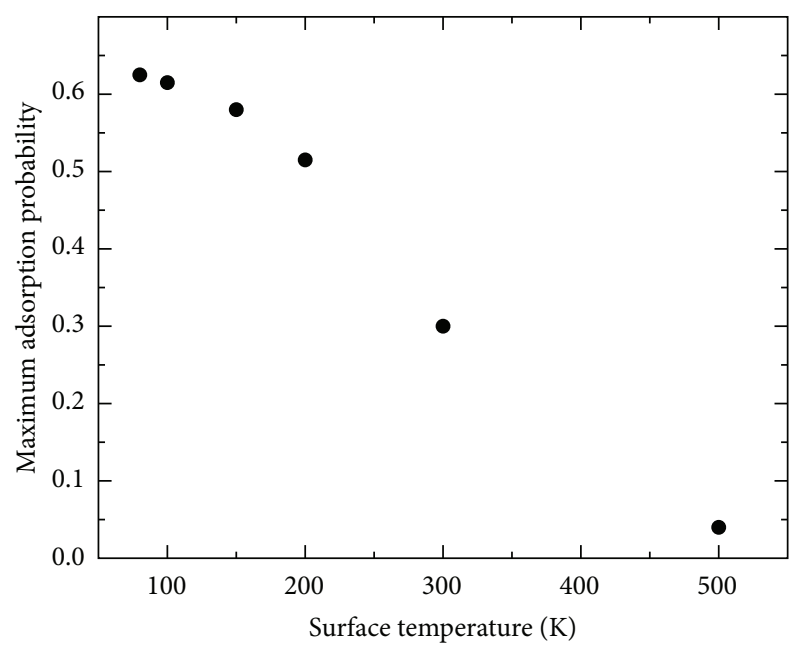

Figure 21: Maximum of the molecular adsorption of $\mathrm{N}_{2}$ on $\mathrm{Fe}(110)$ as a function of the surface temperature. Data taken from [135].

In spite of the big effort already invested by surface scientists, a method to fully take into account the complex motion associated with surface phonons is still to be developed.

6.3. Looking for Accurate Exchange-Correlation Functionals. Also relative to future challenges, it is worthy to mention some of the shortcomings inherent to state-of-the-art DFT simulations. Although a number of exchange-correlation functionals, such as PW91 [158], PBE [159], RPBE [160], or a specific reaction parameter approach applied to them [111] have shown to give good qualitative (and even quantitative) results, in comparison with experiment, for $\mathrm{H}_{2}$ reactive and nonreactive scattering, other molecules present a major challenge. For example, up to now, none of the proposed functionals have been able to yield a sufficiently accurate PES to describe the dissociative adsorption of $\mathrm{O}_{2}$ on $\mathrm{Al}(111)$. For this system, experimental results [161] show a very low dissociation probability at thermal energies, increasing monotonously with the incidence energy, whereas adiabatic molecular dynamics simulations show very high reactivities $[162,163]$ independent of the incidence energy (see Figure 22).

This failure of the standard DFT functionals to accurately describe the interaction of $\mathrm{O}_{2}$ with $\mathrm{Al}(111)$, also observed for adsorption of $\mathrm{O}_{2}$ on $\mathrm{Si}(111)$ [164], is due to the triplet-tosinglet spin conversion, which is not properly described by standard DFT. Contrary to most diatomic molecules, $\mathrm{O}_{2}$ in gas phase (far from the surface) is in its triplet ground state, and when approaching the surface a transition to two oxygen atoms in their spin-singlet state occurs. Aiming to overcome this shortcoming a spin-constrained DFT approach has been proposed $[58,165]$. In this model, the spin of the $\mathrm{O}_{2}$ molecule is constrained to the Hilbert subspace, which prevents spin quenching and charge transfer before the molecule starts interacting with the surface; that is, the molecule is forced to travel in a spin-triplet configuration up to distances close to the surface. It is worth mentioning that an accurate description of triplet-to-singlet spin conversion becomes 


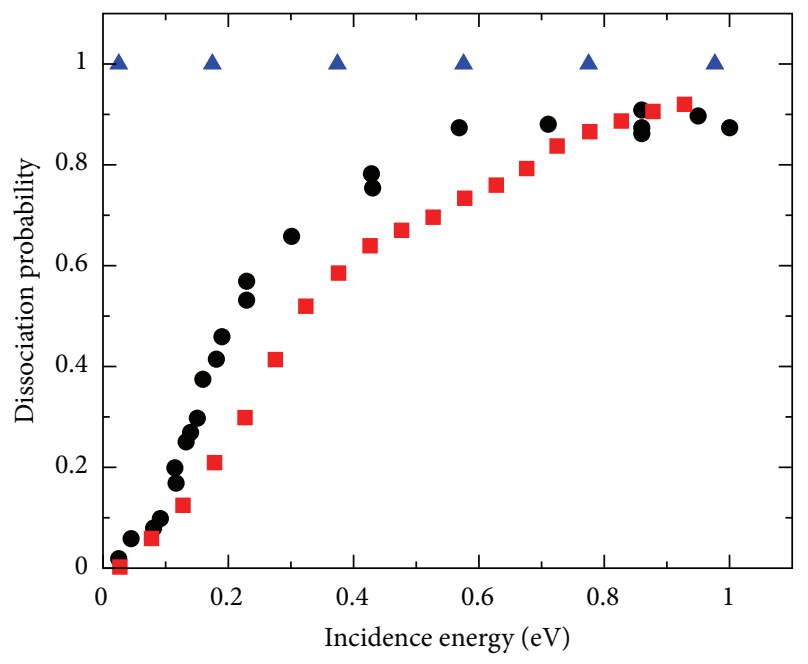

FIGURE 22: Dissociation probability as a function of the incidence energy for $\mathrm{O}_{2}$ on $\mathrm{Al}(111)$. Black solid circles: experimental results from [161]; red solid squares: spin-constrained DFT results from [58]; blue triangles: adiabatic results.

crucial mostly for systems with a low density of states at the Fermi level. For example, standard DFT adiabatic dynamics for $\mathrm{O}_{2} / \mathrm{Ag}(100)$ [45] agrees with experimental data in the absence of dissociative adsorption for energies below $1 \mathrm{eV}$.

Finally, we should mention that standard DFT does not take into account the effect of van der Waals (vdW) interactions. However these forces may play a prominent role for polyatomic molecules interacting with surfaces [166]. Attempts to include vdW forces have been made by Grimme et al. [167-169] using an empirical correction scheme and by Dion et al. [170-172] who proposed to include the vdW forces by expansion to second order in a specific quantity contained in the long range part of the correlation functional. Tkatchenko and Scheffler [173] have proposed a scheme which uses a parameter-free method to define accurately the long range vdW forces from mean-field electronic structure calculations. To conclude, it is also worth mentioning that several efficient implementations of these nonlocal density functionals are already available in commercial codes [174, 175].

\section{Conflict of Interests}

The author declares that there is no conflict of interests regarding the publication of this paper.

\section{Acknowledgment}

The author acknowledges support under MICINN Project FIS2010-25127.

\section{References}

[1] G. Ertl, "Primary steps in catalytic synthesis of ammonia," Journal of Vacuum Science and Technology A: Vacuum, Surfaces and Films, vol. 1, no. 2, pp. 1247-1253, 1983.
[2] A. Groß, "Reactions at surfaces studied by ab initio dynamics calculations," Surface Science Reports, vol. 32, no. 8, pp. 291-340, 1998.

[3] G. Kroes, "Six-dimensional quantum dynamics of dissociative chemisorption of $\mathrm{H}_{2}$ on metal surfaces," Progress in Surface Science, vol. 60, no. 1, pp. 1-5, 1999.

[4] D. Farias and K. H. Rieder, "Atomic beam diffraction from solid surfaces," Reports on Progress in Physics, vol. 61, no. 12, p. 1575, 1998.

[5] D. Farías, H. F. Busnengo, and F. Martín, "Probing reaction dynamics at metal surfaces with $\mathrm{H}_{2}$ diffraction," Journal of Physics: Condensed Matter, vol. 19, Article ID 305003, 2007.

[6] D. Farías and R. Miranda, "Diffraction of molecular hydrogen from metal surfaces," Progress in Surface Science, vol. 86, no. 910, pp. 222-254, 2011.

[7] L. Vattuone, L. Savio, F. Pirani, D. Cappelletti, M. Okada, and M. Rocca, "Interaction of rotationally aligned and of oriented molecules in gas phase and at surfaces," Progress in Surface Science, vol. 85, no. 1-4, pp. 92-160, 2010.

[8] D. A. King and M. G. Wells, "Molecular beam investigation of adsorption kinetics on bulk metal targets: nitrogen on tungsten," Surface Science, vol. 29, no. 2, pp. 454-482, 1972.

[9] C. T. Rettner, L. A. DeLouise, and D. J. Auerbach, "Effect of incidence kinetic energy and surface coverage on the dissociative chemisorption of oxygen on W(110)," The Journal of Chemical Physics, vol. 85, no. 2, pp. 1131-1149, 1986.

[10] G. Comsa, "Angular distribution of scattered and desorbed atoms from specular surfaces," Journal of Chemical Physics, vol. 48, article 3240, no. 7, 1968.

[11] H. A. Michelsen, C. T. Rettner, D. J. Auerbach, and R. N. Zare, "Effect of rotation on the translational and vibrational energy dependence of the dissociative adsorption of $\mathrm{D}_{2}$ on $\mathrm{Cu}(111)$," Journal of Chemical Physics, vol. 98, no. 10, pp. 8294-8307, 1993.

[12] P. Maroni, D. Papageorgopoulos, A. Ruf, R. D. Beck, and T. R. Rizzo, "Efficient stimulated Raman pumping for quantum state resolved surface reactivity measurements," Review of Scientific Instruments, vol. 77, no. 5, Article ID 054103, 2006.

[13] G. O. Sitz, A. C. Kummel, and R. N. Zare, "Direct inelastic scattering of $\mathrm{N}_{2}$ from $\mathrm{Ag}(111)$. I. Rotational populations and alignment," The Journal of Chemical Physics, vol. 89, no. 4, pp. 25582571, 1988.

[14] E. E. Marinero, C. T. Rettner, and R. N. Zare, "Quantumstate-specific detection of molecular hydrogen by three-photon ionization," Physical Review Letters, vol. 48, no. 19, pp. 13231326, 1982.

[15] G. Scoles, Atomics and Molecular Beam Methods, vol. 2, Oxford University Press, New York, NY, USA, 1992.

[16] G. R. Darling and S. Holloway, "The dissociation of diatomic molecules at surfaces," Reports on Progress in Physics, vol. 58, no. 12, pp. 1595-1672, 1995.

[17] G. Kroes and M. F. Somers, "Six-dimensional dynamics of dissociative chemisorption of $\mathrm{H}_{2}$ on metal surfaces," Journal of Theoretical \& Computational Chemistry, vol. 4, no. 2, pp. 493581, 2005.

[18] R. Diez Muino and H. F. Busnengo, Eds., Dynamics of GasSurface Interactions, Springer Series in Surface Science, 2013.

[19] J. E. Lennard-Jones, "Processes of adsorption and diffusion on solid surfaces," Transactions of the Faraday Society, vol. 28, pp. 333-359, 1932.

[20] L. Martin-Gondre, C. Crespos, P. Larregaray, J. C. Rayez, B. van Ootegem, and D. Conte, "Is the LEPS potential accurate 
enough to investigate the dissociation of diatomic molecules on surfaces?" Chemical Physics Letters, vol. 471, no. 1-3, pp. 136-142, 2009.

[21] M. F. Somers, S. M. Kingma, E. Pijper, G. J. Kroes, and D. Lemoine, "Six-dimensional quantum dynamics of scattering of

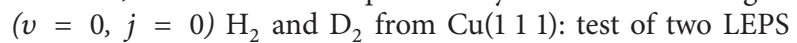
potential energy surfaces," Chemical Physics Letters, vol. 360, no. 3-4, pp. 390-399, 2002.

[22] M. Persson, J. Strömquist, L. Bengtsson, B. Jackson, D. V. Shalashilin, and B. Hammer, "A first-principles potential energy surface for Eley-Rideal reaction dynamics of $\mathrm{H}$ atoms on $\mathrm{Cu}(111)$," Journal of Chemical Physics, vol. 110, no. 4, pp. 22402249, 1999.

[23] S. Caratzoulas, B. Jackson, and M. Persson, "Eley-Rideal and hot-atom reaction dynamics of $\mathrm{H}(\mathrm{g})$ with $\mathrm{H}$ adsorbed on $\mathrm{Cu}(111), " J o u r n a l$ of Chemical Physics, vol. 107, no. 16, pp. 64206431, 1997.

[24] J. Dai and J. Z. H. Zhang, "Quantum adsorption dynamics of a diatomic molecule on surface: four-dimensional fixed-site model for $\mathrm{H}_{2}$ on $\mathrm{Cu}(111)$," The Journal of Chemical Physics, vol. 102, no. 15, pp. 6280-6289, 1995.

[25] A. Forni, G. Wiesenekker, E. J. Baerends, and G. F. Tantardini, "A dynamical study of the chemisorption of molecular hydrogen on the $\mathrm{Cu}(111)$ surface," Journal of Physics: Condensed Matter, vol. 7, no. 36, pp. 7195-7207, 1995.

[26] J. H. McCreery and G. Wolken Jr., "Atomic recombination dynamics on a solid surface: $\mathrm{H}_{2}+\mathrm{W}(001)$," Journal of Chemical Physics, vol. 64, no. 7, pp. 2845-2853, 1976.

[27] G. Wiesenekker, "An analytical six-dimensional potential energy surface for dissociation of molecular hydrogen on $\mathrm{Cu}(100)$," Journal of Chemical Physics, vol. 104, no. 18, pp. 73447358, 1996.

[28] A. Gross, S. Wilke, and M. Scheffler, "Six-dimensional quantum dynamics of adsorption and desorption of $\mathrm{H}_{2}$ at $\mathrm{Pd}(100)$ : steering and steric effects," Physical Review Letters, vol. 75, no. 14, Article ID 2718, 1995.

[29] T. Ho and H. Rabitz, "A general method for constructing multidimensional molecular potential energy surfaces from $a b$ initio calculations," The Journal of Chemical Physics, vol. 104, no. 7, pp. 2584-2597, 1996.

[30] D. E. Makarov and H. Metiu, "Fitting potential-energy surfaces: a search in the function space by directed genetic programming," Journal of Chemical Physics, vol. 108, no. 2, pp. 590-598, 1998.

[31] H. F. Busnengo, A. Salin, and W. Dong, "Representation of the $6 \mathrm{D}$ potential energy surface for a diatomic molecule near a solid surface," The Journal of Chemical Physics, vol. 112, no. 17, pp. 7641-7651, 2000.

[32] C. Crespos, M. A. Collins, E. Pijper, and G. J. Kroes, "Multidimensional potential energy surface determination by modified Shepard interpolation for a molecule-surface reaction: $\mathrm{H}_{2}$ + Pt(1 11 1)," Chemical Physics Letters, vol. 376, no. 5-6, pp. 566575, 2003.

[33] T. J. Frankcombe, M. A. Collins, and D. H. Zhang, "Modified Shepard interpolation of gas-surface potential energy surfaces with strict plane group symmetry and translational periodicity," Journal of Chemical Physics, vol. 137, no. 14, Article ID 144701, 2012.

[34] S. Lorenz, A. Groß, and M. Scheffler, "Representing highdimensional potential-energy surfaces for reactions at surfaces by neural networks," Chemical Physics Letters, vol. 395, no. 4-6, pp. 210-215, 2004.
[35] G. Kresse, "Dissociation and sticking of $\mathrm{H}_{2}$ on the $\mathrm{Ni}(111),(100)$, and (110) substrate," Physical Review B, vol. 62, no. 12, pp. 8295$8305,2000$.

[36] R. A. Olsen, H. F. Busnengo, A. Salin, M. F. Somers, G. J. Kroes, and E. J. Baerends, "Constructing accurate potential energy surfaces for a diatomic molecule interacting with a solid surface: $\mathrm{H}_{2}+\mathrm{Pt}(111)$ and $\mathrm{H}_{2}+\mathrm{Cu}(100)$," The Journal of Chemical Physics, vol. 116, no. 9, pp. 3841-3855, 2002.

[37] M. A. Di Césare, H. F. Busnengo, W. Dong, and A. Salin, "Role of dynamic trapping in $\mathrm{H}_{2}$ dissociation and reflection on $\mathrm{Pd}$ surfaces," Journal of Chemical Physics, vol. 118, no. 24, pp. 1122611234, 2003.

[38] G. Volpilhac and A. Salin, "Dissociative adsorption of N2 on the W(1 00 ) surface," Surface Science, vol. 556, no. 2-3, pp. 129-144, 2004.

[39] P. Riviére, H. F. Busnengo, and F. Martín, "Density functional theory study of $\mathrm{H}$ and $\mathrm{H}_{2}$ interacting with $\mathrm{NiAl}(110), "$ The Journal of Chemical Physics, vol. 121, p. 751, 2004.

[40] M. Luppi, D. A. McCormack, R. A. Olsen, and E. J. Baerends, "Rotational effects in the dissociative adsorption of $\mathrm{H}_{2}$ on the $\mathrm{Pt}$ (211) stepped surface," Journal of Chemical Physics, vol. 123, no. 16, Article ID 164702, 2005.

[41] J. K. Vincent, R. A. Olsen, G. J. Kroes, M. Luppi, and E. J. Baerends, "Six-dimensional quantum dynamics of dissociative chemisorption of $\mathrm{H}_{2}$ on $\mathrm{Ru}(0001)$," The Journal of Chemical Physics, vol. 122, no. 4, Article ID 044701, 2005.

[42] A. Salin, "Theoretical study of hydrogen dissociative adsorption on the $\mathrm{Cu}(110)$ surface," Journal of Chemical Physics, vol. 124, no. 10, Article ID 104704, 2006.

[43] M. Alducin, R. Díez Muiño, H. F. Busnengo, and A. Salin, "Why $\mathrm{N}_{2}$ molecules with thermal energy abundantly dissociate on W(100) and not on W(110)," Physical Review Letters, vol. 97, no. 5, Article ID 056102, 2006.

[44] H. F. Busnengo and A. E. Martínez, " $\mathrm{H}_{2}$ chemisorption on W(100) and W(110) surfaces," Journal of Physical Chemistry C, vol. 112, no. 14, pp. 5579-5588, 2008.

[45] M. Alducin, H. F. Busnengo, and R. Díez Muiño, "Dissociative dynamics of spin-triplet and spin-singlet $\mathrm{O}_{2}$ on $\mathrm{Ag}(100)$ ), The Journal of Chemical Physics, vol. 129, no. 22, Article ID 224702, 2008.

[46] G. Laurent, F. Martín, and H. F. Busnengo, "Theoretical study of hydrogen dissociative adsorption on strained pseudomorphic monolayers of $\mathrm{Cu}$ and Pd deposited onto a $\mathrm{Ru}(0001)$ substrate," Physical Chemistry Chemical Physics, vol. 11, no. 33, pp. 73037311, 2009.

[47] A. Lozano, A. Gross, and H. F. Busnengo, "Adsorption dynamics of $\mathrm{H}_{2}$ on $\mathrm{Pd}(100)$ from first principles," Physical Chemistry Chemical Physics, vol. 11, no. 27, pp. 5814-5822, 2009.

[48] C. Díaz, R. A. Olsen, H. F. Busnengo, and G. J. Kroes, "Dynamics on six-dimensional potential energy surfaces for $\mathrm{H}_{2} / \mathrm{Cu}(111)$ : corrugation reducing procedure versus modified shepard interpolation method and PW91 versus RPBE," Journal of Physical Chemistry C, vol. 114, no. 25, pp. 11192-11201, 2010.

[49] J. C. Chen, M. Ramos, C. Arasa et al., "Dynamics of $\mathrm{H}_{2}$ dissociation on the $1 / 2 \mathrm{ML} c(2 \times 2)-\mathrm{Ti} / \mathrm{Al}(100)$ surface," Physical Chemistry Chemical Physics, vol. 14, no. 9, pp. 3234-3247, 2012.

[50] J. Ischtwan and M. A. Collins, "Molecular potential energy surfaces by interpolation," The Journal of Chemical Physics, vol. 100, no. 11, pp. 8080-8088, 1994.

[51] D. H. Zhang, M. A. Collins, and S.-Y. Lee, "First-principles theory for the $\mathrm{H}+\mathrm{H}_{2} \mathrm{O}, \mathrm{D}_{2} \mathrm{O}$ reactions," Science, vol. 290, no. 5493, pp. 961-963, 2000. 
[52] C. Crespos, M. A. Collins, E. Pijper, and G. J. Kroes, "Application of the modified Shepard interpolation method to the determination of the potential energy surface for a molecule-surface reaction: $H_{2}+\mathrm{Pt}(111)$," The Journal of Chemical Physics, vol. 120, no. 5, pp. 2392-2404, 2004.

[53] R. van Harrevelt, K. Honkala, J. K. Nørskov, and U. Manthe, "The reaction rate for dissociative adsorption of $\mathrm{N}_{2}$ on stepped $\mathrm{Ru}(0001)$ : six-dimensional quantum calculations," Journal of Chemical Physics, vol. 122, no. 23, Article ID 234702, 2005.

[54] C. Díaz, J. K. Vincent, G. P. Krishnamohan et al., "Multidimensional effects on dissociation of $\mathrm{N}_{2}$ on $\mathrm{Ru}(0001)$," Physical Review Letters, vol. 96, no. 9, Article ID 096102, 2006.

[55] I. M. N. Groot, J. C. Juanes-Marcos, C. Díaz, M. F. Somers, R. A. Olsen, and G. Kroes, "Dynamics of dissociative adsorption of hydrogen on a CO-precovered $\mathrm{Ru}(0001)$ surface: a comparison of theoretical and experimental results," Physical Chemistry Chemical Physics, vol. 12, no. 6, pp. 1331-1340, 2010.

[56] P. N. Abufager, C. Crespos, and H. F. Busnengo, "Modified Shepard interpolation method applied to trapping mediated adsorption dynamics," Physical Chemistry Chemical Physics, vol. 9, no. 18, pp. 2258-2265, 2007.

[57] T. J. Frankcombe and M. A. Collins, "Growing fragmented potentials for gas-surface reactions: The reaction between hydrogen atoms and hydrogen-terminated silicon (111)," Journal of Physical Chemistry C, vol. 116, no. 14, pp. 7793-7802, 2012.

[58] J. Behler, B. Delley, S. Lorenz, K. Reuter, and M. Scheffler, "Dissociation of $\mathrm{O}_{2}$ at $\mathrm{Al}(111)$ : the role of spin selection rules," Physical Review Letters, vol. 94, no. 3, Article ID 036104, 2005.

[59] I. Goikoetxea, J. Beltrán, J. Meyer, J. I. Juaristi, M. Alducin, and K. Reuter, "Non-adiabatic effects during the dissociative adsorption of $\mathrm{O}_{2}$ at $\mathrm{Ag}(111)$ ? A first-principles divide and conquer study," New Journal of Physics, vol. 14, Article ID 013050, 2012.

[60] J. Ludwig and D. G. Vlachos, "Ab initio molecular dynamics of hydrogen dissociation on metal surfaces using neural networks and novelty sampling," Journal of Chemical Physics, vol. 127, no. 15, Article ID 154716, 2007.

[61] J. Dai and J. C. Light, "Six dimensional quantum dynamics study for dissociative adsorption of $\mathrm{H}_{2}$ on $\mathrm{Cu}(111)$ surface," Journal of Chemical Physics, vol. 107, no. 5, pp. 1676-1679, 1997.

[62] E. Pijper, G. J. Kroes, R. A. Olsen, and E. J. Baerends, “Reactive and diffractive scattering of $\mathrm{H}_{2}$ from $\mathrm{Pt}(111)$ studied using a sixdimensional wave packet method," Journal of Chemical Physics, vol. 117, no. 12, pp. 5885-5898, 2002.

[63] C. Crespos, H.-D. Meyer, R. C. Mowrey, and G. J. Kroes, "Multiconfiguration time-dependent Hartree method applied to molecular dissociation on surfaces: $\mathrm{H}_{2}+\mathrm{Pt}(111)$," Journal of Chemical Physics, vol. 124, no. 7, Article ID 074706, 2006.

[64] M. H. Beck, A. Jäckle, G. A. Worth, and H. D. Meyer, “The multiconfiguration time-dependent Hartree (MCTDH) method: a highly efficient algorithm for propagating wavepackets," Physics Report, vol. 324, no. 1, pp. 1-105, 2000.

[65] A. S. Muzas, J. I. Juaristi, M. Alducin, R. D. Muio, G. J. Kroes, and C. Díaz, "Vibrational deexcitation and rotational excitation of $\mathrm{H}_{2}$ and $\mathrm{D}_{2}$ scattered from $\mathrm{Cu}(111)$ : adiabatic versus nonadiabatic dynamics," The Journal of Chemical Physics, vol. 137, no. 6, Article ID 064707, 2012.

[66] H. F. Busnengo, W. Dong, P. Sautet, and A. Salin, "Surface temperature dependence of rotational excitation of $\mathrm{H}_{2}$ scattered from Pd(111)," Physical Review Letters, vol. 87, Article ID 127601, 2001.
[67] C. Díaz, M. F. Somers, G. J. Kroes, H. F. Busnengo, A. Salin, and F. Martín, "Quantum and classical dynamics of $\mathrm{H}_{2}$ scattering from Pd(111) at off-normal incidence," Physical Review B, vol. 72, Article ID 035401, 2005.

[68] C. Díaz, H. F. Busnengo, P. Rivière et al., "A classical dynamics method for $\mathrm{H}_{2}$ diffraction from metal surfaces," The Journal of Chemical Physics, vol. 122, no. 15, Article ID 154706, 2005.

[69] C. Díaz, F. Martín, G. J. Kroes, M. Minniti, D. Farías, and R. Miranda, " $\mathrm{H}_{2}$ diffraction from a strained pseudomorphic monolayer of $\mathrm{Cu}$ deposited on $\mathrm{Ru}$ (0001)," The Journal of Physical Chemistry C, vol. 116, p. 13671, 2012.

[70] B. Jackson and H. Metiu, "The dynamics of $\mathrm{H}_{2}$ dissociation on $\mathrm{Ni}(100)$ : a quantum mechanical study of a restricted twodimensional model," The Journal of Chemical Physics, vol. 86, no. 2, pp. 1026-1035, 1986.

[71] M. R. Hand and S. Holloway, "A theoretical study of the dissociation of $\mathrm{H}_{2} / \mathrm{Cu}$," The Journal of Chemical Physics, vol. 91, no. 11, pp. 7209-7219, 1989.

[72] J. Sheng and J. Z. H. Zhang, "Dissociative chemisorption of $\mathrm{H}_{2}$ on Ni surface: time-dependent quantum dynamics calculation and comparison with experiment," The Journal of Chemical Physics, vol. 96, no. 5, pp. 3866-3874, 1992.

[73] R. C. Mowrey, "Dissociative adsorption of $\mathrm{H}_{2}$ using the closecoupling wave packet method," The Journal of Chemical Physics, vol. 99, no. 9, pp. 7049-7055, 1993.

[74] G. R. Darling and S. Holloway, "Angular and vibrational effects in the sticking and scattering of $\mathrm{H}_{2}$," The Journal of Chemical Physics, vol. 97, no. 7, pp. 5182-5192, 1992.

[75] J. Dai and J. Z. H. Zhang, "Steric effect in dissociative chemisorption of hydrogen on Cu," Surface Science, vol. 319, no. 1-2, pp. 193-198, 1994.

[76] A. Gross, "The role of lateral surface corrugation for the quantum dynamics of dissociative adsorption and associative desorption," The Journal of Chemical Physics, vol. 102, no. 12, pp. 5045-5058, 1995.

[77] G. J. Kroes, G. Wiesenekker, E. J. Baerends, and R. C. Mowrey, "Competition between vibrational excitation and dissociation in collisions of $\mathrm{H}_{2}$ with $\mathrm{Cu}(100)$," Physical Review B, vol. 53, no. 15, pp. 10397-10401, 1996.

[78] A. Gross, B. Hammer, M. Scheffler, and W. Brenig, "Highdimensional quantum dynamics of adsorption and desorption of $\mathrm{H}_{2}$ at $\mathrm{Cu}(111)$," Physical Review Letters, vol. 73, no. 23, pp. 31213124, 1994.

[79] G. R. Darling and S. Holloway, "Rotational motion and the dissociation of $\mathrm{H}_{2}$ on $\mathrm{Cu}(111)$," The Journal of Chemical Physics, vol. 101, no. 4, pp. 3268-3281, 1994.

[80] R. C. Mowrey, G. J. Kroes, and E. J. Baerends, "Dissociative adsorption of $\mathrm{H}_{2}$ on $\mathrm{Cu}(100)$ : fixed-site calculations for impact at hollow and top sites," Journal of Chemical Physics, vol. 108, no. 16, pp. 6906-6915, 1998.

[81] G. J. Kroes, E. J. Baerends, and R. C. Mowrey, "Six-dimensional quantum dynamics of dissociative chemisorption of $(v=0, j=$ 0) $\mathrm{H}_{2}$ on $\mathrm{Cu}(100)$," Physical Review Letters, vol. 78, no. 18, pp. 3583-3586, 1997.

[82] G. J. Kroes, E. J. Baerends, and R. C. Mowrey, "Six-dimensional quantum dynamics of dissociative chemisorption of $(v=0, j=$ 0) $\mathrm{H}_{2}$ on $\mathrm{Cu}(100)$," Physical Review Letters, vol. 78, article 3583, 1997.

[83] H. F. Busnengo, C. Crespos, W. Dong, A. Salin, and J. C. Rayez, "Role of orientational forces in nonactivated molecular dissociation on a metal surface," Physical Review B, vol. 63, no. 4, Article ID 041402, 2001. 
[84] C. Crespos, H. F. Busnengo, W. Dong, and A. Salin, "Analysis of $\mathrm{H}_{2}$ dissociation dynamics on the Pd(111) surface," Journal of Chemical Physics, vol. 114, no. 24, pp. 10954-10962, 2001.

[85] G. Anger, A. Winkler, and K. D. Rendulic, "Adsorption and desorption kinetics in the systems $\mathrm{H}_{2} / \mathrm{Cu}(111), \mathrm{H}_{2} / \mathrm{Cu}(110)$ and $\mathrm{H}_{2} / \mathrm{Cu}(100)$," Surface Science, vol. 220, no. 1, pp. 1-17, 1989.

[86] H. F. Berger, M. Leisch, A. Winkler, and K. D. Rendulic, "A search for vibrational contributions to the activated adsorption of $\mathrm{H}_{2}$ on copper," Chemical Physics Letters, vol. 175, no. 5, pp. 425-428, 1990.

[87] H. A. Michelsen and D. J. Auerbach, "A critical examination of data on the dissociative adsorption and associative desorption of hydrogen at copper surfaces," The Journal of Chemical Physics, vol. 94, no. 11, pp. 7502-7520, 1991.

[88] H. A. Michelsen, C. T. Rettner, and D. J. Auerbach, "Statespecific dynamics of $\mathrm{D}_{2}$ desorption from $\mathrm{Cu}(111)$ : the role of molecular rotational motion in activated adsorption-desorption dynamics," Physical Review Letters, vol. 69, no. 18, pp. 26782681, 1992.

[89] C. T. Rettner, D. J. Auerbach, and H. A. Michelsen, "Role of vibrational and translational energy in the activated dissociative adsorption of $\mathrm{D}_{2}$ on $\mathrm{Cu}(111)$," Physical Review Letters, vol. 68, no. 8, pp. 1164-1167, 1992.

[90] C. T. Rettner, D. J. Auerbach, and H. A. Michelsen, "Observation of direct vibrational excitation in collisions of $\mathrm{H}_{2}$ and $\mathrm{D}_{2}$ with a $\mathrm{Cu}(111)$ surface," Physical Review Letters, vol. 68, no. 16, pp. 2547-2550, 1992.

[91] C. T. Rettner, H. A. Michelsen, and D. J. Auerbach, "Determination of quantum-state-specific gas-surface energy transfer and adsorption probabilities as a function of kinetic energy," Chemical Physics, vol. 175, no. 1, pp. 157-169, 1993.

[92] C. T. Rettner, H. A. Michelsen, and D. J. Auerbach, "Quantumstate-specific dynamics of the dissociative adsorption and associative desorption of $\mathrm{H}_{2}$ at a $\mathrm{Cu}(111)$ surface," The Journal of Chemical Physics, vol. 102, no. 11, pp. 4625-4641, 1995.

[93] H. Hou, S. J. Guiding, C. T. Rettner, A. M. Wodtke, and D. J. Auerbach, "The stereodynamics of a gas-surface reaction," Science, vol. 277, no. 5322, pp. 80-82, 1997.

[94] A. Hodgson, J. Moryl, P. Traversaro, and H. Zhao, "Energy transfer and vibrational effects in the dissociation and scattering of $\mathrm{D}_{2}$ from $\mathrm{Cu}(111)$," Nature, vol. 356, no. 6369, pp. 501-504, 1992.

[95] A. Hodgson, P. Samson, A. Wight, and C. Cottrell, "Rotational excitation and vibrational relaxation of $\mathrm{H}_{2}(\mathrm{v}=1, \mathrm{~J}=0)$ scattered from Cu(111)," Physical Review Letters, vol. 78, no. 5, pp. 963966, 1997.

[96] M. J. Murphy and A. Hodgson, "Adsorption and desorption dynamics of $\mathrm{H}_{2}$ and $\mathrm{D}_{2}$ on $\mathrm{Cu}(111)$ : the role of surface temperature and evidence for corrugation of the dissociation barrier," Journal of Chemical Physics, vol. 108, no. 10, pp. 4199-4211, 1998.

[97] D. Wetzig, M. Rutkowski, R. David, and H. Zacharias, "Rotational corrugation in associative desorption of $\mathrm{D}_{2}$ from $\mathrm{Cu}(111)$," Europhysics Letters, vol. 36, no. 1, pp. 31-36, 1996.

[98] S. J. Gulding, A. M. Wadtke, H. Hou, C. T. Rettner, H. A. Michelsen, and D. J. Auerbach, "Alignment of $\mathrm{D}_{2}(\mathrm{v}, \mathrm{J})$ desorpbed from $\mathrm{Cu}(111)$ : low sensitivity of activated dissociative chemisorption to approach geometry," The Journal of Chemical Physics, vol. 105, p. 9702, 1996.

[99] G. R. Darling and S. Holloway, "Dissociation thresholds and the vibrational excitation process in the scattering of $\mathrm{H}_{2}$," Surface Science, vol. 307-309, pp. 153-158, 1994.
[100] J. Dai and J. C. Light, “The steric effect in a full dimensional quantum dynamics simulation for the dissociative adsorption of $\mathrm{H}_{2}$ on $\mathrm{Cu}(111)$," The Journal of Chemical Physics, vol. 108, no. 18, pp. 7816-7820, 1998.

[101] J. Dai, J. Sheng, and J. Z. H. Zhang, "Symmetry and rotational orientation effects in dissociative adsorption of diatomic molecules on metals: $\mathrm{H}_{2}$ and $\mathrm{HD}$ on $\mathrm{Cu}(111)$," The Journal of Chemical Physics, vol. 101, no. 2, pp. 1555-1563, 1994.

[102] G. R. Darling and S. Holloway, "Translation-to-vibrational excitation in the dissociative adsorption of $\mathrm{D}_{2}$," The Journal of Chemical Physics, vol. 97, no. 1, pp. 734-736, 1992.

[103] J. Sheng and J. Z. H. Zhang, "Quantum dynamics studies of adsorption and desorption of hydrogen at a $\mathrm{Cu}(111)$ surface," The Journal of Chemical Physics, vol. 99, no. 2, pp. 1373-1381, 1993.

[104] C. Díaz, R. A. Olsen, D. J. Auerbach, and G. J. Kroes, "Sixdimensional dynamics study of reactive and non reactive scattering of $\mathrm{H}_{2}$ from $\mathrm{Cu}(111)$ using a chemically accurate potential energy surface," Physical Chemistry Chemical Physics, vol. 12, no. 24, pp. 6499-6519, 2010.

[105] G. J. Kroes, E. Pijper, and A. Salin, "Dissociative chemisorption of $\mathrm{H}_{2}$ on the $\mathrm{Cu}(110)$ surface: a quantum and quasiclassical dynamical study," Journal of Chemical Physics, vol. 127, no. 16, Article ID 164722, 2007.

[106] F. Nattino, C. Díaz, B. Jackson, and G. Kroes, "Effect of surface motion on the rotational quadrupole alignment parameter of $\mathrm{D}_{2}$ reacting on $\mathrm{Cu}(111)$," Physical Review Letters, vol. 108, no. 23, Article ID 236104, 2012.

[107] P. S. Thomas, M. F. Somers, A. W. Hoekstra, and G. J. Kroes, "Chebyshev high-dimensional model representation (Chebyshev-HDMR) potentials: application to reactive scattering of $\mathrm{H}_{2}$ from $\mathrm{Pt}(111)$ and $\mathrm{Cu}(111)$ surfaces," Physical Chemistry Chemical Physics, vol. 14, no. 24, pp. 8628-8643, 2012.

[108] A. Marashdeh, S. Casolo, L. Sementa, H. Zacharias, and G. J. Kroes, "Surface temperature effects on dissociative chemisorption of $\mathrm{H}_{2}$ on $\mathrm{Cu}(100)$," Journal of Physical Chemistry C, vol. 117, no. 17, pp. 8851-8863, 2013.

[109] L. Sementa, M. Wijzenbroek, B. J. Van Kolck et al., "Reactive scattering of $\mathrm{H}_{2}$ from $\mathrm{Cu}(100)$ : comparison of dynamics calculations based on the specific reaction parameter approach to density functional theory with experiment," Journal of Chemical Physics, vol. 138, no. 4, Article ID 044708, 2013.

[110] B. Hammer, M. Scheffler, K. W. Jacobsen, and J. K. Nørskov, "Multidimensional potential energy surface for $\mathrm{H}_{2}$ dissociation over Cu(111)," Physical Review Letters, vol. 73, no. 10, pp. 14001403, 1994.

[111] C. Díaz, E. Pijper, R. A. Olsen, H. F. Busnengo, D. J. Auerbach, and G. J. Kroes, "Chemically accurate simulation of a prototypical surface reaction: $\mathrm{H}_{2}$ dissociation on $\mathrm{Cu}(111)$," Science, vol. 326, no. 5954, pp. 832-834, 2009.

[112] U. Nielsen, D. Halstead, S. Holloway, and J. K. Nørskov, “The dissociative adsorption of hydrogen: two-, three-, and fourdimensional quantum simulations," The Journal of Chemical Physics, vol. 93, no. 4, pp. 2879-2884, 1990.

[113] S. Nave, D. Lemoine, M. F. Somers, S. M. Kingma, and G. J. Kroes, "Six-dimensional quantum dynamics of $(v=0, j=0) \mathrm{D}_{2}$ and of $(v=1, j=0) \mathrm{H}_{2}$ scattering form $\mathrm{Cu}(111)$," The Journal of Chemical Physics, vol. 122, Article ID 214709, 2005.

[114] C. Díaz, A. Perrier, and G. J. Kroes, "Associative desorption of $\mathrm{N}_{2}$ from $\mathrm{Ru}\left(\begin{array}{l}0 \\ 0\end{array} 0\right.$ 1): a computational study," Chemical Physics Letters, vol. 434, no. 4-6, pp. 231-236, 2007. 
[115] E. Watts and G. O. Sitz, "State-to-state scattering in a reactive system: $\mathrm{H}_{2}(\mathrm{v}=1, \mathrm{~J}=1)$ from $\mathrm{Cu}(111)$," Journal of Chemical Physics, vol. 114, no. 9, pp. 4171-4179, 2001.

[116] L. C. Shackman and G. O. Sitz, "State-to-state scattering of $\mathrm{D}_{2}$ from $\mathrm{Cu}(100)$ and $\mathrm{Pd}(111)$, , The Journal of Chemical Physics, vol. 123, no. 6, Article ID 064712, 2005.

[117] G. Kroes, C. Díaz, E. Pijper, R. A. Olsen, and D. J. Auerbach, "Apparent failure of the Born-Oppenheimer static surface model for vibrational excitation of molecular hydrogen on copper," Proceedings of the National Academy of Sciences of the United States of America, vol. 107, no. 49, pp. 20881-20886, 2010.

[118] C. Díaz, H. F. Busnengo, F. Martin, and A. Salin, "Angular distribution of $\mathrm{H}_{2}$ molecules scattered from the $\mathrm{Pd}(111)$ surface," The Journal of Chemical Physics, vol. 118, no. 6, p. 2886, 2003.

[119] C. Díaz, F. Martín, H. F. Busnengo, and A. Salin, “Theoretical analysis of the relation between $\mathrm{H}_{2}$ dissociation and reflection on Pd surfaces," The Journal of Chemical Physics, vol. 120, no. 1, pp. 321-328, 2004.

[120] G. Comsa and R. David, "Dynamical parameters of desorbing molecules," Surface Science Reports, vol. 5, no. 4, pp. 145-198, 1985.

[121] P. Nieto, E. Pijper, D. Barredo et al., "Reactive and nonreactive scattering of $\mathrm{H}_{2}$ from a metal surface is electronically adiabatic," Science, vol. 312, no. 5770, pp. 86-89, 2006.

[122] B. Gumhalter, "Single- and multiphonon atom-surface scattering in the quantum regime," Physics Report, vol. 351, no. 1-2, pp. $1-159,2001$.

[123] D. Farías, C. Díaz, P. Rivíere et al., "In-plane and out-of-plane diffraction of $\mathrm{H}_{2}$ from metal surface," Physical Review Letters, vol. 93, no. 24, Article ID 246104, 2004.

[124] G. Laurent, D. Barredo, D. Farías et al., "Experimental and theoretical study of rotationally inelastic diffraction of $\mathrm{D}_{2}$ from NiAl(110)," Physical Chemistry Chemical Physics, vol. 12, no. 43, pp. 14501-14507, 2010.

[125] D. Barredo, G. Laurent, C. Díaz et al., "Experimental evidence of dynamic trapping in the scattering of $\mathrm{H}_{2}$ from $\mathrm{Pd}(110)$," The Journal of Chemical Physics, vol. 125, no. 5, Article ID 051101, 2006.

[126] B. Gergen, H. Nienhaus, W. H. Weinberg, and E. W. McFarland, "Chemically induced electronic excitations at metal surfaces," Science, vol. 294, no. 5551, pp. 2521-2523, 2001.

[127] A. M. Wodtke, J. C. Tully, and D. J. Auerbach, "Electronically non-adiabatic interactions of molecules at metal surfaces: Can we trust the Born-Oppenheimer approximation for surface chemistry?" International Reviews in Physical Chemistry, vol. 23, no. 4, pp. 513-539, 2004.

[128] J. D. White, J. Chen, D. Matsiev, D. J. Auerbach, and A. M. Wodtke, "Conversion of large-amplitude vibration to electron excitation at a metal surface," Nature, vol. 433, no. 7025, pp. 503505, 2005.

[129] G. Sitz, "Surface chemistry: approximate challenges," Nature, vol. 433, no. 7025, p. 470, 2005.

[130] G. Kroes, "Frontiers in surface scattering simulations," Science, vol. 321, no. 5890, pp. 794-797, 2008.

[131] Y. Huang, C. T. Rettner, D. J. Auerbach, and A. M. Wodtke, "Vibrational promotion of electron transfer," Science, vol. 290, no. 5489, pp. 111-114, 2000.

[132] N. Shenvi, S. Roy, and J. C. Tully, "Dynamical steering and electronic excitation in NO scattering from a gold surface," Science, vol. 326, no. 5954, pp. 829-832, 2009.
[133] J. Meyer and K. Reuter, "Electron-hole pairs during the adsorption dynamics of $\mathrm{O}_{2}$ on $\mathrm{Pd}(100)$ : Exciting or not?" New Journal of Physics, vol. 13, Article ID 085010, 2011.

[134] C. Díaz, J. K. Vincent, G. P. Krishnamohan et al., "Reactive and nonreactive scattering of $N_{2}$ from $\mathrm{Ru}(0001)$ : a six-dimensional adiabatic study," Journal of Chemical Physics, vol. 125, Article ID 114706, 2006.

[135] I. Goikoetxea, M. Alducin, E. Díez-Muiño, and J. I. Juaristi, "Dissociative and non-dissociative adsorption dynamics of $\mathrm{N}_{2}$ on Fe(110)," Physical Chemistry Chemical Physics, vol. 14, p. 7471, 2012.

[136] L. Martin-Gondre, M. Alducin, G. A. Bocan, R. Díez Muiño, and J. I. Juaristi, "Competition between electron and phonons excitation in the scattering of nitrogen atoms and moleculesb off tungsten and silver metal surface," Physical Review Letters, vol. 108, Article ID 096101, 2012.

[137] X. J. Shen, A. Lozano, W. Dong, H. F. Busnengo, and X. H. Yan, "Towards bond selective chemistry from first principles: methane on metal surfaces," Physical Review Letters, vol. 112, Article ID 046101, 2014.

[138] R. R. Smith, D. R. Killelea, D. F. DelSesto, and A. L. Utz, "Preference for vibrational over translational energy in a gassurface reaction," Science, vol. 304, no. 5673, pp. 992-995, 2004.

[139] L. B. F. Juurlink, R. R. Smith, D. R. Killelea, and A. L. Utz, "Comparative study of $\mathrm{C}-\mathrm{H}$ stretch and bend vibrations in methane activation on $\mathrm{Ni}(100)$ and $\mathrm{Ni}(111), "$ Physical Review Letters, vol. 94, Article ID 208303, 2005.

[140] R. D. Beck, P. Maroni, D. C. Papageorgopoulos, T. T. Dang, M. P. Schmid, and T. R. Rizzo, "Vibrational mode-specific reaction of methane on a nickel surface," Science, vol. 302, no. 5642, pp. 98-100, 2003.

[141] S. Nave and B. Jackson, "Methane dissociation on Ni(111): the role of lattice reconstruction," Physical Review Letters, vol. 98, no. 17, Article ID 173003, 2007.

[142] S. Nave and B. Jackson, "Vibrational mode-selective chemistry: methane dissociation on Ni(100)," Physical Review B, vol. 81, no. 23, Article ID 233408, 2010.

[143] A. K. Tiwari, S. Nave, and B. Jackson, "Methane dissociation on Ni(111): a new understanding of the lattice effect," Physical Review Letters, vol. 103, no. 25, Article ID 253201, 2009.

[144] L. Romm, G. Katz, R. Kosloff, and M. Asscher, "Vibrational and kinetic energy: molecular beam experiments and quantum mechanical calculations," The Journal of Physical Chemistry B, vol. 101, p. 2213, 1997.

[145] L. Diekhöner, H. Mortensen, A. Baurichter, E. Jensen, V. V. Petrunin, and A. C. Luntz, " $N 2$ dissociative adsorption on $\mathrm{Ru}(0001)$ : the role of energy loss," Journal of Chemical Physics, vol. 115, no. 19, pp. 9028-9035, 2001.

[146] A. C. Luntz, M. Persson, and G. O. Sitz, "Theoretical evidence for nonadiabatic vibrational deexcitation in $\mathrm{H}_{2}\left(\mathrm{D}_{2}\right)$ state-tostate scattering from $\mathrm{Cu}(100)$," Journal of Chemical Physics, vol. 124, no. 9, Article ID 091101, 2006.

[147] J. W. Gadzuk and H. Metiu, "Theory of electron-hole pair excitations in unimolecular processes at metal surfaces. I. X-ray edge effects," Physical Review B, vol. 22, no. 6, pp. 2603-2613, 1980.

[148] H. Metiu and J. W. Gadzuk, "Theory of rate processes at metal surfaces. II. the role of substrate electronic excitations," The Journal of Chemical Physics, vol. 74, no. 4, pp. 2641-2653, 1981.

[149] B. Gumhalter and S. G. Davison, "Effect of electronic relaxation on covalent adsorption reaction rates," Physical Review B, vol. 30, no. 6, pp. 3179-3190, 1984. 
[150] J. I. Juaristi, M. Alducin, R. Díez-Muiño, H. F. Busnengo, and A. Salin, "Role of electron-hole pair excitations in the dissociative adsorption of diatomic molecules on metal surfaces," Physical Review Letters, vol. 100, no. 11, Article ID 116102, 2008.

[151] N. Shenvi, S. Roy, and J. C. Tully, "Nonadiabatic dynamics at metal surfaces: Independent-electron surface hopping," Journal of Chemical Physics, vol. 130, no. 17, Article ID 174107, 2009.

[152] N. H. Nahler, J. D. White, J. LaRue, D. J. Auerbach, and A. M. Wodtke, "Inverse velocity dependence of vibrationally promoted electron emission from a metal surface," Science, vol. 321, no. 5893, pp. 1191-1194, 2008.

[153] M. Hand and J. Harris, "Recoil effects in surface dissociation," The Journal of Chemical Physics, vol. 92, no. 12, pp. 7610-7617, 1990.

[154] A. Groß and A. Dianat, "Hydrogen dissociation dynamics on precovered Pd surfaces: langmuir is still right," Physical Review Letters, vol. 98, no. 20, Article ID 206107, 2007.

[155] J. C. Tully, "Dynamics of gas-surface interactions: 3D generalized Langevin model applied to fcc and bcc surfaces," The Journal of Chemical Physics, vol. 73, no. 4, pp. 1975-1985, 1980.

[156] H. F. Busnengo, M. A. Di Césare, W. Dong, and A. Salin, "Surface temperature effects in dynamic trapping mediated adsorption of light molecules on metal surfaces: $\mathrm{H}_{2}$ on $\mathrm{Pd}(111)$ and Pd(110)," Physical Review B, vol. 72, no. 12, Article ID 125411, 2005.

[157] M. Bonfanti, M. F. Somers, C. Díaz, H. F. Busnengo, and G. J. Kroes, "7D Quantum dynamics of $\mathrm{H}_{2}$ scattering from $\mathrm{Cu}(111)$ : the accuracy of the phonon sudden approximation," Zeitschrift für Physikalische Chemie, vol. 227, pp. 1397-1420, 2013.

[158] J. P. Perdew, J. A. Chevary, S. H. Vosko et al., "Atoms, molecules, solids, and surfaces: Applications of the generalized gradient approximation for exchange and correlation," Physical Review $B$, vol. 46, no. 11, pp. 6671-6687, 1992.

[159] J. P. Perdew, K. Burke, and M. Ernzerhof, "Generalized gradient approximation made simple," Physical Review Letters, vol. 77, no. 18, pp. 3865-3868, 1996.

[160] B. Hammer, L. B. Hansen, and J. K. Nørskov, "Improved adsorption energetics within density-functional theory using revised Perdew-Burke-Ernzerhof functionals," Physical Review B, vol. 59, no. 11, pp. 7413-7421, 1999.

[161] L. Österlund, I. Zoric, and B. Kasemo, "Dissociative sticking of $\mathrm{O}_{2}$ on Al(111)," Physical Review B, vol. 55, Article ID 15452, 1997.

[162] K. Honkala and K. Laasonen, "Oxygen molecule dissociation on the $\mathrm{Al}(111)$ surface," Physical Review Letters, vol. 84, no. 4, pp. 705-708, 2000.

[163] Y. Yourdshahyan, B. Razaznejad, and B. I. Lundqvist, "Adiabatic potential-energy surfaces for oxygen on $\mathrm{Al}(111)$," Physical Review B, vol. 65, no. 7, Article ID 075416, 2002.

[164] K. Kato, T. Uda, and K. Terakura, "Backbond oxidation of the $\mathrm{Si}(001)$ surface: narrow channel of barrierless oxidation," Physical Review Letters, vol. 80, no. 9, pp. 2000-2003, 1998.

[165] J. Behler, K. Reuter, and M. Scheffler, "Nonadiabatic effects in the dissociation of oxygen molecules at the $\mathrm{Al}(111)$ surface," Physical Review B-Condensed Matter and Materials Physics, vol. 77, no. 11, Article ID 115421, 2008.

[166] P. Lazić, N. Atodiresei, V. Caciuc, R. Brako, B. Gumhalter, and S. Blügel, "Rationale for switching to nonlocal functionals in density functional theory," Journal of Physics Condensed Matter, vol. 24, Article ID 424215, 2012.

[167] S. Grimme, "Accurate description of van der Waals complexes by density functional theory including empirical corrections,"
Journal of Computational Chemistry, vol. 25, no. 12, pp. 14631473,2004

[168] S. Grimme, "Semiempirical GGA-type density functional constructed with a long-range dispersion correction," Journal of Computational Chemistry, vol. 27, no. 15, pp. 1787-1799, 2006.

[169] S. Grimme, J. Antony, S. Ehrlich, and H. Krieg, "A consistent and accurate ab initio parametrization of density functional dispersion correction (DFT-D) for the 94 elements H-Pu," Journal of Chemical Physics, vol. 132, no. 15, Article ID 154104, 2010.

[170] M. Dion, H. Rydberg, E. Schröder, D. C. Langreth, and B. I. Lundqvist, "Van der Waals density functional for general geometries," Physical Review Letters, vol. 92, no. 24, Article ID 246401, 2004.

[171] M. Dion, H. Rydberg, E. Schröder, D. C. Langreth, and B. I. Lundqvist, "Erratum: Van der Waals density functional for general geometries," Physical Review Letters, vol. 95, Article ID 109902, 2005.

[172] J. Klime, D. R. Bowler, and A. Michaelides, "Van der Waals density functionals applied to solids," Physical Review B, vol. 83, no. 19, Article ID 195131, 2011.

[173] A. Tkatchenko and M. Scheffler, "Accurate molecular van der Waals interactions from ground-state electron density and freeatom reference data," Physical Review Letters, vol. 102, no. 7, Article ID 073005, 2009.

[174] P. Lazić, N. Atodiresei, M. Alaei, V. Caciuc, S. Blügel, and R. Brako, "JuNoLo-Jülich nonlocal code for parallel post-processing evaluation of vdW-DF correlation energy," Computer Physics Communications, vol. 181, no. 2, pp. 371-379, 2010.

[175] G. Román-Pérez and J. M. Soler, "Efficient implementation of a van der Waals density functional: application to double-wall carbon nanotubes," Physical Review Letters, vol. 103, Article ID 096102, 2009.

[176] K. D. Rendulic, G. Anger, and A. Winkler, "Wide range nozzle beam adsorption data for the systems $\mathrm{H}_{2} /$ nickel and $\mathrm{H}_{2} / \mathrm{Pd}(100)$," Surface Science, vol. 208, no. 3, pp. 404-424, 1989.

[177] E. Pijper, M. F. Somers, G. J. Kroes et al., "Six-dimensional quantum dynamics of scattering of $(v=0, j=0) \mathrm{H}_{2}$ from $\operatorname{Pt}\left(\begin{array}{lll}1 & 1 & 1\end{array}\right)$ : comparison to experiment and to classical dynamics results," Chemical Physics Letters, vol. 347, no. 4-6, pp. 277-284, 2001.

[178] G. Laurent, C. Díaz, H. F. Busnengo, and F. Martín, "Nonmonotonic dissociative adsorption of vibrationally excited $\mathrm{H}_{2}$ on metal surface," Physical Review B, vol. 81, Article ID 161404, p. 81, 2010.

[179] P. Rivière, M. F. Somers, G. J. Kroes, and F. Martín, "Quantum dynamical study of the $\mathrm{H}_{2}$ and $\mathrm{D}_{2}$ dissociative adsorption and diffraction from the NiAl(110) alloy surface," Physical Review B, vol. 73, Article ID 205417, 2006. 

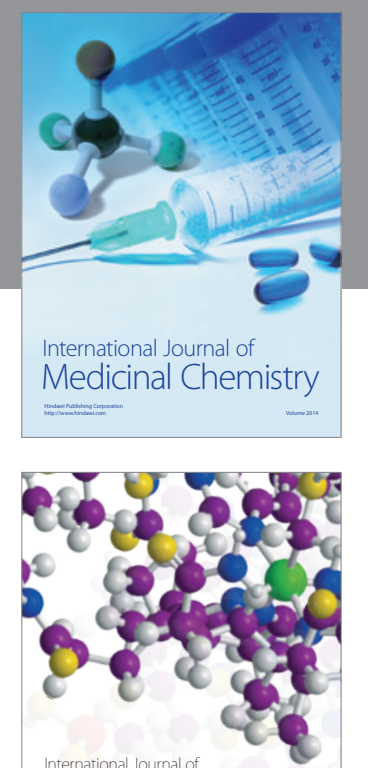

\section{Carbohydrate} Chemistry

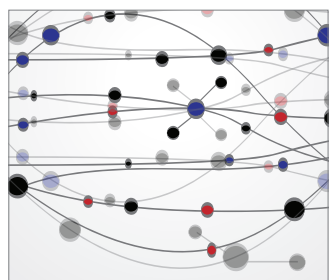

The Scientific World Journal
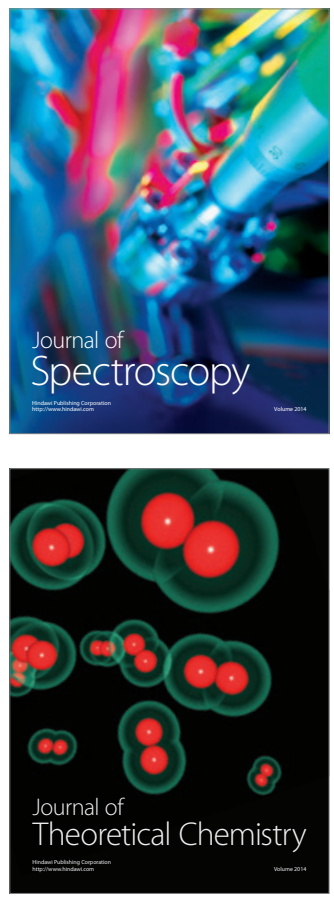
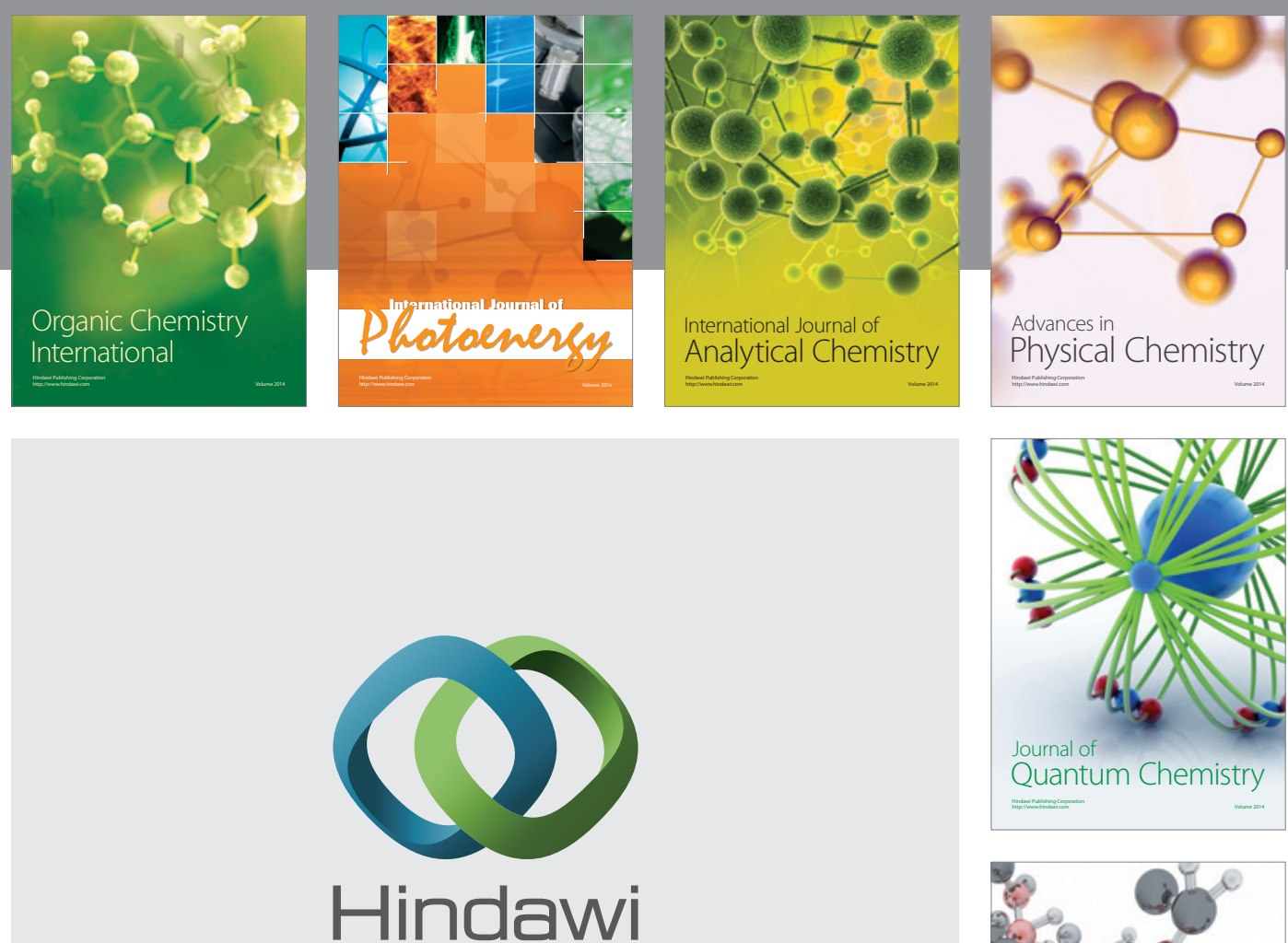

Submit your manuscripts at

http://www.hindawi.com

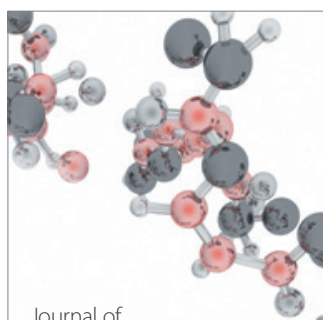

Analytical Methods

in Chemistry

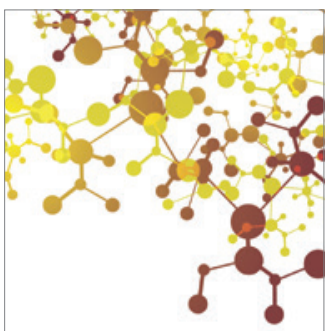

Journal of

Applied Chemistry

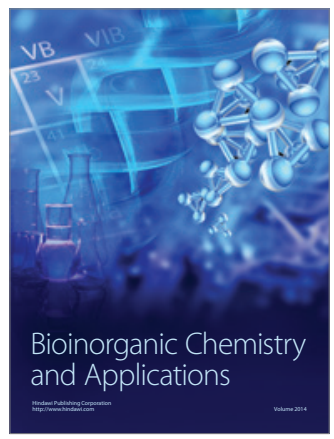

Inorganic Chemistry
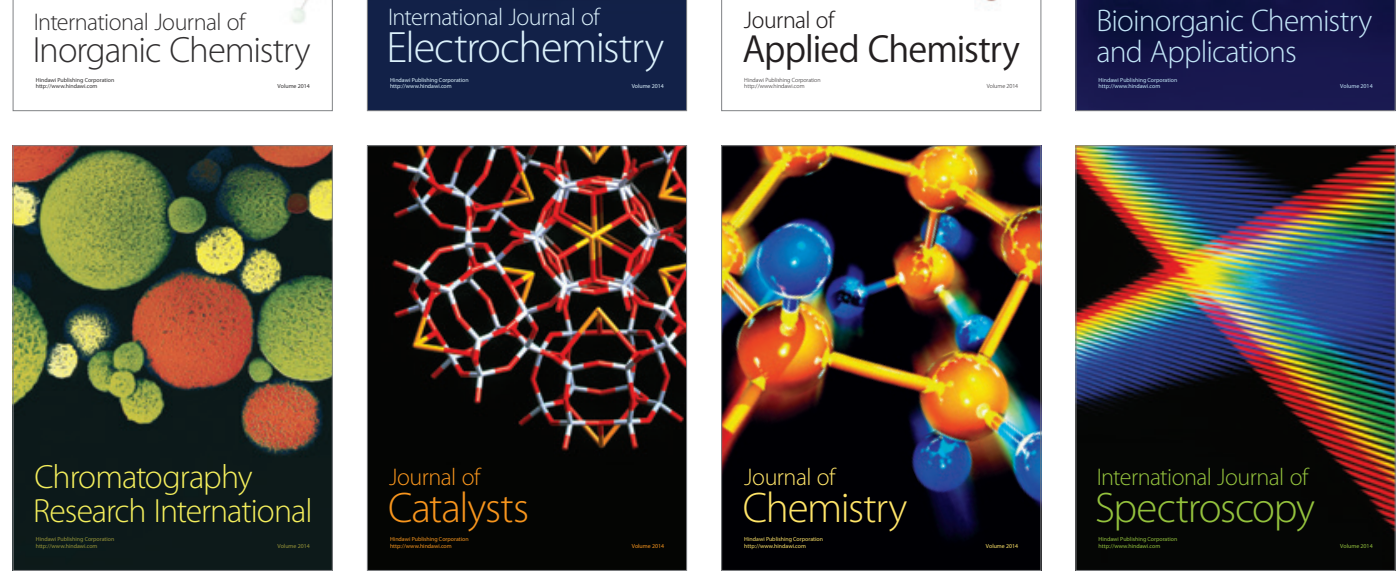